Vol.18,\#8, Junio 2010

http://revista-redes.rediris.es

\title{
La globalización de la cultura y la cuestión de la diversidad cultural: estudio de los flujos mundiales de traducciones entre 1979 y 2002
}

\author{
Germain Barré - Université Paris Dauphine - IRISSO ${ }^{1}$
}

\begin{abstract}
Resumen
El artículo plantea un cuestionamiento a la diversidad cultural mediante el estudio de los flujos de traducciones entre 1979 y 2002. Con la ayuda del análisis de redes o método "estructural", se utilizan indicadores para medir la diversidad cultural y evaluar el reconocimiento de las diferentes lenguas. Por ejemplo, se observa una clara caída de la diversidad de los intercambios de traducción a partir de 1989.

Gracias a este método, se comprende también la evolución de la estructura de flujos de traducciones $y$, por consecuente, la dinámica de estos flujos. De este modo, es posible constatar que la estructura de flujos de traducciones resulta estable en los años 80: se oponen en torno al inglés y al ruso dos grandes grupos de lenguas. Luego de la caída del muro de Berlín las relaciones preferenciales de traducción entre las lenguas oficiales de los antiguos países comunistas desaparecen, mientras que la centralidad del inglés se vuelve más importante. De manera general, la elección del idioma de las obras que serán traducidas se funda, antes que nada, sobre consideraciones geográficas y políticas.
\end{abstract}

En síntesis, el análisis de redes aporta otra visión de la globalización. De este modo, nos parece importante que herramientas como ésta sean integradas en futuros estudios relacionados con esta problemática.

Palabras clave: Globalización - Cultura - Diversidad Cultural - Traducciones - Análisis de Redes Sociales

\section{Abstract}

The article presents a challenge to cultural diversity through the study of translation flows between 1979 and 2002. With the help of network analysis or method "structural" indicators are used to measure cultural diversity and assess the recognition of different languages. For example, there is a clear decline in the diversity of exchange translation from 1989.

Using this method, we also understand the changing structure translation flows and, consequently, the dynamics of these flows. Thus, it is possible when the structure flows translations are stable in the 80's: around opposing English and Russian languages, two major groups. After the fall of the Berlin Wall preferential translation relations between the official languages of the former communist countries disappear, while the centrality of English becomes more important. In general, the choice of language works to be translated is based, above all, on geographical and political considerations.

In summary, network analysis provides a different vision of globalization. Thus, it seems important that tools like this to be integrated in future studies related to this issue.

Keywords: Globalization - Culture - Cultural Diversity - Translation - Social Network Analysis.

\footnotetext{
${ }^{1}$ Enviar correspondencia a: barre.germain@gmail.com miembro del Observatorio de Redes Intra- e Interorganizacionales (ORIO; Centre for Research on Intra- and Inter-Organisational Networks).
}

\section{Agradecimientos}

Quisiera agradecer especialmente a Catherine Comet, a Ainhoa de Federico y a Abram De Swaan por sus comentarios. El autor agradece a Felipe Valdivieso la traducción del artículo. 


\section{Traducciones y diversidad cultural}

\section{Traducciones, cultura y Estados}

La traducción es "una práctica muy antigua a la cual hay que recurrir debido a la multiplicidad de lenguas. Henri van Hoof, en su Historia de la traducción en Occidente, señala su existencia desde el Antiguo Imperio (2640-2040 AC)". (Cachin y Bruyère, 2002; Pág. 506). Con la formación de los Estados-Naciones, las lenguas se fueron codificando de manera progresiva: la actividad de traducción pasó entonces a relacionarse con las nuevas relaciones (de conflicto y de cooperación) entre los Estados (Heilbron, 1999) ${ }^{2}$.

En particular, la traducción permite el contacto entre dos culturas (la transmisión de un mensaje), pero también es el reflejo de la apertura de una cultura a otra (una forma de comprensión del otro): "[la traducción] refleja las fluctuaciones del interés dado a las culturas extranjeras según los períodos y las naciones. Ella pone en evidencia los abismos culturales muchas veces difíciles de superar, señala los países refractarios a la introducción de culturas extranjeras. La importancia y el número de traducciones en la producción editorial de un país en una época dada son, entonces, indicadores significativos de su apertura a otras culturas" (Cachin y Bruyère, 2002, Págs. 506-507). Marie-Françoise Cachin y Claire Bruyère citan a Lieven d'Hulst, autor de Cien años de teoría francesa de la traducción, quien concibe la traducción como un "documento-clave sobre la forma como el extranjero - o el extraño- es definido, asimilado o rechazado"3.

Sin embargo, como lo señala Lévi-Strauss, la relación entre lenguaje y cultura es una de las más complicadas: la lengua y la cultura están estrechamente ligadas en una relación de interdependencia. El lenguaje es a la vez un producto de la cultura (la refleja), una parte de ésta (un elemento entre otros), al mismo tiempo que la condiciona, ya que el individuo adquiere la cultura de su grupo mediante el lenguaje (Lévi-Strauss, 1958). Así, una traducción necesita a menudo una adaptación, una aclimatación que busca reducir las distancias culturales entre país de origen y país de acogida. Por esto la retórica, ciertos esquemas de argumentación o ciertas palabras plantean problemas de traducción: es el caso de la traducción de la palabra inglesa people, al francés peuple (pueblo), cargada de referencias políticas a finales del siglo XVIII, o bien de la traducción del léxico freudiano. 


\section{Cuatro ejes de estudio de la diversidad cultural}

En la idea de cultura esta incluida la idea de diversidad, al contrario de la naturaleza que es siempre y en todas partes la misma (haciendo abstracción del problema de la evolución biológica). La cuestión de la diversidad cultural resulta compleja, pudiéndose distinguir cuatro ejes en el estudio de los bienes culturales.

Un premier eje consiste en estudiar la diversidad de los intercambios de bienes culturales (1). Así, según un autor de la Escuela de Francfort, "la libertad humana no se mide según la opción ofrecida al individuo, el único factor decisivo para determinar [la libertad humana] es lo que el individuo puede elegir y elige" (Marcuse, 1968, Págs. 32$33)^{4}$.

En el caso de los bienes culturales, "lo que el individuo puede elegir" depende de la diversidad de productores (países o empresas) y de la producción de bienes culturales (2). Un artículo disponible en el sitio de la UNESCO revela tasas elevadas de concentración en el comercio internacional de bienes culturales que no parecen haber evolucionado mucho durante los años 90. Por ejemplo, en 1990 Japón, Estados Unidos, Alemania y Gran Bretaña representan el 55,4\% del total de las exportaciones de bienes culturales en el mundo y el $47 \%$ de las importaciones fueron realizadas por Estados Unidos, Alemania, Gran Bretaña y Francia ${ }^{5}$. Los autores concluyen que la mayor parte de estos intercambios se efectúan entre un número reducido de países sin dar mayores precisiones. Estas cifras muestran que el crecimiento del comercio internacional de bienes culturales no parece ligado a una mayor diversificación de países productores de bienes culturales ${ }^{6}$. Estos fenómenos de concentración han sido igualmente observados a nivel de las empresas productoras de bienes culturales, así como a nivel de la difusión-

2 Heilbron (2001) recuerda el lazo histórico entre cultura y Estado: « Un centro cultural unido a un Estado poderoso y centralizado se sobreponía, poco a poco, a los centros relacionados con una ciudad o federación. Hacia 1700, Francia e Inglaterra podían contar con una ascendencia real sobre los otros Estados y formas estatales; es también en estos países donde se vieron nacer instituciones culturales ligadas a un aparato estatal creciente, instituciones llamadas a formar los cimientos de su cultura nacional"

3 Citado por Cachin y Bruyère, 2002, página 506.

${ }^{4}$ A propósito de la problemática de la estandarización de la producción de bienes culturales, ver también Adorno T.W. et Horkheimer M. (1974 [1947]).

5 Ver la rúbrica «questions» del sitio Internet de la UNESCO : $\underline{\text { http://portal.unesco.org/culture/fr/ev.php }}$

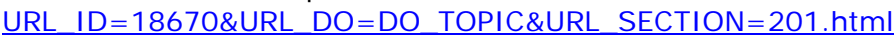

6 Esta concentración se produce en circunstancias que el comercio internacional de bienes culturales se ha cuadruplicado en los últimos dos decenios. Entre 1980 y 1998, los intercambios comerciales relativos a libros y revistas, música, artes plásticas, cine, fotografía, radio, televisión, juegos y artículos deportivos han aumentado de 95 à 388 billones de dólares. Este aumento concierne también las ventas de grabaciones sonoras (27.000 millones de ventas en 1990, 38.671 millones de dólares en 1998). 
distribución ${ }^{7}$.

Por otro lado, "lo que elige el individuo" puede relacionarse con el comportamiento de otros individuos. En efecto, la oferta de bienes culturales puede diversificarse, al mismo tiempo que se compran los mismos "productos" (lo que se manifestaría en la compra extendida de bests-sellers). De esta manera, la diversidad de elecciones de los consumidores (3) constituye un tercer eje de estudio de la diversidad cultural.

Finalmente, podemos agregar la recepción de la obra cultural (4) (entendida aquí como la interacción entre el texto y el lector) como el cuarto y último eje del estudio de la diversidad cultural. Esta recepción puede engendrar fenómenos de "criollización", de reapropiación y de adaptación de elementos de otra cultura. Los filósofos de la Escuela de Constanza han buscado comprender el proceso de interacción texto-lector. Podemos citar en particular a Wolfgang, quien analizó "el efecto del texto", proceso que va del texto al lector. El texto contiene "blancos" que suscitan el trabajo imaginario del lector ${ }^{8}$ y su cooperación activa. Para Hans Robert Gauss, la manera como el lector se apropia del texto y lo interpreta depende de sus expectativas literarias (que dependen a su vez, especialmente, de sus lecturas anteriores), de sus expectativas concretas, de códigos socioculturales, de valores, de normas, de gustos que constituyen el universo del lector y los niveles de competencias específicas adquiridas (nivel de formación, conocimientos adquiridos en la institución escolar y/o en el medio familiar). Junto con lo anterior, diversos elementos de un libro coaccionan su lectura: códigos, valores, referencias comunes a los textos y al lector que orientan su lectura, así como la lengua o el estilo. Por añadidura, el texto se inscribe en un momento de la historia literaria y pertenece a un género que obedece a códigos que orientan y condicionan la lectura ${ }^{9}$ : la obra actúa sobre los horizontes de expectativas del público

\footnotetext{
7 Ver por ejemplo el artículo de Arnault de Saint-Ange sobre el mundo de la edición en Francia Le monde de l'édition 2003-2005. La fin d'une époque, disponible en la dirección: http://www.lektiecriture.com/contrefeux/Le-monde-de-l-edition-2003-2005. html. Ver también Schiffrin (1999).

8 Por ejemplo, investigadores han podido observar interpretaciones diversas de un mismo libro durante la primera lectura realizada por individuos pertenecientes a un mismo grupo social (Burgos, 1992), en dos épocas diferentes (el libro La educación sentimental de Flaubert es percibido de manera diferente antes y después de 1968 por el público estudiantil [Goulemont, 1985] : antes, el libro se resume al relato de los amores de un adolescente y una mujer madura; después, Federico es percibido como un burgués reaccionario y cobarde que prefiere una aventura galante a la acción revolucionaria), o bien en dos países diferentes (el estudio de Leenhardt (1982) sobre la recepción en Francia y Hungría de dos obras que describen las sociedades de los dos países en los años 60: Les choses de George Pérec y Le cimetière de rouille de Fejes).

9 El prefacio, los diversos consejos (incluyendo los de los periodistas y críticos), los prólogos, etc., limitan como un freno las derivas imaginarias del lector (Jouve, 1993). La dimensión material del libro orienta su lectura (tapa y contratapa, tipografía, compaginación, ilustraciones) (Chartier, 1993; Mc Kenzie, 1981). La presentación de los dos principales autores de la Escuela de Constanza así como estos ejemplos son extraídos de Horellou Lafarge \& Segré (2003), con excepción de las obras de Goulemont et McKenzie citadas por Roger Chartier (1985, dir.).
} 
Debemos precisar que la diversidad de elecciones de los consumidores y la recepción de la obra cultural no son hechos de individuos aislados de otros individuos: sus relaciones tienen igualmente una incidencia sobre la recepción que dan a las obras culturales. Además, los cuatro ejes antes citados pueden estar relacionados entre sí superponiéndose múltiples dimensiones (sociológica, económica, política, simbólica, psicológica... etc.). En el caso de los intercambios culturales transnacionales, este tipo de enfoque permite escapar del economicismo y del culturalismo (Bourdieu, 1979; Heilbron, 1999) ${ }^{10}$.

\section{Nuestros dos ejes de estudio: la diversidad de intercambios de bienes culturales en el caso de las traducciones y el estudio de la dinámica de los flujos de traducciones entre 1979 y 2002}

Mientras que el segundo eje de la diversidad cultural ha sido estudiado extensamente, la cuestión sobre la diversidad de los intercambios de bienes culturales continúa siendo poco explorada. Las traducciones a nivel internacional entre 1972 y 2002 constituirán nuestro objeto de estudio. Nuestro interés se centrará en la problemática de la diversidad cultural para lo cual someteremos a prueba, en particular, la hipótesis de la concentración de flujos de traducciones durante este período. Enseguida, intentaremos explicar la evolución de las dinámicas de flujos de traducciones en dos etapas. En una primera instancia, mostraremos la importancia del sentido de las traducciones y mediremos el reconocimiento de los idiomas con la ayuda de un nuevo indicador.

En una segunda instancia, se describirá de forma detallada la dinámica de los flujos mundiales de traducciones. Este trabajo constituye lo esencial de este texto, cuyo carácter es decididamente exploratorio. Considerando el carácter innovador de nuestro enfoque, se han buscado más bien obtener medidas y realizar constataciones, antes que inscribirse en un cuadro teórico preciso.

\footnotetext{
10 Bourdieu, 1979, página 1: “Existe una economía de bienes culturales, pero dicha economía tiene una lógica especifica que hay que extraer para escapar del economicismo. Esto, primero trabajando en establecer las condiciones en las cuáles son producidos los consumidores de bienes culturales y su gusto, al mismo tiempo que describiendo las diferentes maneras de apropiarse de aquellos bienes que en un momento dado son considerados como obras de arte y de las condiciones sociales de la constitución del modo de apropiación que es considerado legítimo".

Heilbron (1999): «Instead of conceiving the cultural realm as merely derivative of global economic structures, it is more fruitful to view transnational cultural exchange as a relatively autonomous sphere, as an international arena with economic, political and symbolic dimensions. (...) Such a view of avoids both the economism of certain varieties of world systems theory and the culturalism which trends to prevail in cultural studies ».
} 


\section{Metodología}

\section{Los “ miembros" de la red}

"Ayudar a reconocer las propiedades estructurales de los conjuntos sociales es una de las contribuciones de la sociología. Para esto, el método llamado "estructural" parte de la observación de relaciones y de la ausencia de relaciones entre los miembros de un conjunto social. A partir de estas constataciones, este método busca reconstituir un sistema de relaciones y describir la influencia de dicho sistema sobre el comportamiento de sus miembros. Siendo los sistemas de relaciones sociales habitualmente complejos, esta metodología los representa de manera simplificada, deliberadamente reductora, pero práctica para la comprensión y la explicación. En su lenguaje, una "estructura" es entonces una representación simplificada de un sistema social complejo. Esta simplificación permite identificar regularidades en la composición y la distribución de las relaciones" (Lazega, 1999, Pág. 3) ${ }^{11}$.

En nuestro estudio, los "miembros" de la red son las lenguas vivas traducidas al menos una vez entre 1979 y 2002, siendo las traducciones las "relaciones" entre estos "miembros" 12. El Index Translationum, que registra los flujos internacionales de traducciones, nos sirvió como base de datos. Esta fuente parece ser bastante completa (volveremos a tocar este punto más adelante) y se encuentra disponible en el sitio Internet de la UNESCO ${ }^{13}$.

Con el fin de crear matrices distinguimos, en un primer momento, las lenguas vivas de otras lenguas dentro del conjunto de lenguas traducidas entre 1979 y 2002. Las principales lenguas vivas figuran en un archivo que distingue más de 16000 categorías de población, categorías establecidas a partir del lugar donde residen dichas poblaciones, su lengua, religión, etc. ${ }^{14}$. Ciertas lenguas no figuran en este archivo. Entre éstas se encuentran las lenguas muertas (76 idiomas como el latín, el griego antiguo y el

\footnotetext{
${ }^{11}$ En una perspectiva estructuralista, el método denominado Análisis de Redes incorpora una teoría de la acción individual: el individuo actúa en un contexto ya estructurado que le ofrece oportunidades y limitantes. En ocasiones participa a la emergencia o al reforzamiento de estas estructuras. En nuestro estudio esta teoría de la acción individual es evidentemente dejada a un lado, ya que una lengua no « actúa ».

12 Se tomaron en cuenta todos los «tipos» de libros. Las siguientes cifras son las proporciones que representa cada « campo» en promedio anual hasta 1989 y después de 1992. El campo literario representa el 49\% de las traducciones durante los dos períodos, las ciencias "duras" el16\%. La rúbrica "Derecho, Ciencias Sociales y Educación" representa el $11 \%$ de las traducciones hasta 1989 y el $8 \%$ después de 1992. Las otras cuatro categorías ("Historia, Geografía, Biografías", "Religión, teología”, “Filosofía, psicología” y "Artes, Juegos, Deportes") representan cada una entre el 5 y el $7 \%$ de las traducciones durante los dos períodos considerados. La estabilidad de estos porcentajes nos lleva a suponer que el tipo de obras traducidas no es responsable de los cambios acontecidos respecto al reconocimiento de las lenguas entre 1979 et 2002.

${ }^{13}$ http://portal.unesco.org/culture/fr/ev.php URL_ID=7810\&URL_DO=DO_TOPIC\&URL_SECTION=201.html
} 
sánscrito), las lenguas "construidas" (3, entre las que destaca el esperanto), pero también lenguas vivas que no portan el mismo nombre en los datos del Index Translationum de la UNESCO y en el otro archivo citado ${ }^{15}$. Únicamente las lenguas vivas fueron conservadas en nuestras matrices.

A continuación suprimimos las traducciones concernientes a varias de las lenguas no especificadas $^{16}$, así como los dialectos cuyo número de traducciones resulta insignificante en relación a la lengua de la cual surgieron dichos dialectos ${ }^{17}$. Para los otros dialectos, se realizaron agrupamientos con la lengua de la cual surgieron ${ }^{18}$.

Utilizando esta base de datos, creamos una matriz que representa el flujo de traducciones entre las 665 lenguas vivientes para cada año del período que va entre 1979 y 2002

La fiabilidad de los datos del Index Translationum debe ser tomada con precaución. Por una parte, la definición de un libro varía de un país al otro: por ejemplo los libros escolares o los documentos administrativos, pueden o no corresponder a esta definición según el país considerado. Por otra parte, la fiabilidad de los datos es variable de un país a otro y de un año a otro. Hemos intentado mejorar esto en una treintena de países para los cuales los datos nos parecían poco fiables en uno o más años, reemplazando los datos poco fiables por datos mas recientes que parecen más confiables ${ }^{19}$. Estas

14 Este archivo se encuentra disponible en el sitio http://www.joshuaproject.net bajo el nombre « jpallpeoplesbyctry » y figura en la lista de documentos relativos a la globalización recopilada por Miguel Angel Centeno. Aprovecho este espacio para agradecerle que haya puesto a disposición esta lista.

15 Por ejemplo, «Filipino / Pilipino » (que figura bajo esta etiqueta en el Index Translationum) y «Tagalog » (que figura bajo esta etiqueta en el archivo citado más arriba) son en realidad la misma lengua. Esta distinción fue realizada a partir de los sitios: www.wikipedia.com y www.ethnologue.com

${ }^{16}$ Agrupadas bajo la etiqueta « Múltiples lenguajes».

17 «Croatian, dialects of »; «English, dialects of »; «French, dialects of »; «German, dialects of »; «Spanish, dialects of »; «Tamil, dialects of »; « Uighur, dialects of ». Comorian; Ngazidja » (estos dos dialectos son aquellos de las dos principales Islas Comores, pero que no se distinguen del comorano en el archivo que poseemos) ; « Dakota \& Dakota, dialects of »; « Dargwa \& Dargwa, dialects of »; « Franco-Provençal \& Franco-Provençal dialects of »; « Kala Lagaw Ya \& Kala Lagaw Ya, dialects of »; « Khakas \& Khakas, dialects of »; « Maya, Yucatán \& Maya, Yucatán, dialects of »; « Mongo \& Mongo, dialects of »; «Picard \& Picard, dialects of »; «Romani, Vlax \& Romani, Vlax, dialects of »; « Schwyzerdütsch $\&$ Schwyzerdütsch, other dialects of $»$. Hemos verificado de manera cuidadosa si las traducciones de una obra en estos dialectos no eran idénticas a una traducción de esa misma obra en la lengua de la cual surge el dialecto. Un ejemplo ficticio: una obra escrita en inglés, traducida en picarda y en un dialecto picardés el mismo año. En ese caso, no habríamos contabilizado la traducción en el "dialecto" picardés.

19 Cuando el número de traducciones de un año a otro varia de forma anormal (acercándose a menudo a una casi ausencia de datos), se modificaron los datos respetando la regla del último año fiable conservado para los siguientes países: Arabia Saudita, Argentina, Australia, Bangladesh, Bulgaria, Colombia, República de Corea, Egipto, Finlandia, Grecia, India, Indonesia, Islandia, Italia, J ordania, Malasia, Myanmar, Nueva-Zelanda, Reino Unido, Federación Rusa, Sri Lanka, Suecia, Siria, Tailandia, Turquía, Ucrania, Vaticano y Yugoslavia. Chile, Holanda e Irán representan casos excepcionales, ya que los datos de los años 1985 (para los dos primeros países) y de 1986 (para Irán) fueron reemplazados por datos de los años precedentes, a falta de datos fiables disponibles antes de dichas fechas. 
debilidades subrayan la dificultad de comparar datos a nivel internacional. Sin embargo, los datos entregados por el Index Translationum son los únicos disponibles a nivel internacional.

\title{
2. “Lengua original “ y "lengua objetivo"
}

De aquí en adelante utilizaremos el término "lengua original" para designar el idioma en el cual una obra fue escrita. El término "lengua objetivo" designa la lengua en la cual se es traducida una obra. Un ejemplo extraído de la traducción de la La veuve Couderc de Georges Simenon en 1982 :

\author{
Lengua Original \\ Lengua objetivo \\ Francés (La veuve Couderc) \\ Alemán (Die Witwe Couderc)
}

Cronológicamente, esta obra fue escrita primero en francés para luego ser traducida al alemán. En análisis de redes, el sentido del flujo es importante: se distinguen las elecciones "emitidas" y las elecciones "recibidas". En este sentido, consideramos que la lengua escogida es la lengua original, lo que en el ejemplo precedente se traduce como: Alemán $\rightarrow$ Francés (donde $\rightarrow$ significa "elegida")

Así, una relación existe si al menos una traducción es realizada desde una lengua original A (inglés, por ejemplo) hacia una lengua objetivo B (i.e. francés). Si se cuenta al menos con una traducción de la lengua original B (francés) a la lengua objetivo A (inglés), se contabiliza otra relación.

\section{I . Resultados}

\section{La concentración de flujos de traducción entre 1979 y 2002}

A nivel mundial, el número de traducciones aumenta cada año desde 1989 (con la excepción de los años 2000 y 2002), pasando en promedio de 50.000 en 1989 a más de

\footnotetext{
Los datos de Reino Unido fueron reemplazados a partir del año 1990 por los datos del año 1989. Siendo el inglés la lengua más reconocida a nivel internacional, las traducciones del Reino Unido representan una importancia menor en el reconocimiento de esta lengua.

Ningún dato ha sido entregado por Irán entre 1988 y 1998. No obstante, las traducciones de este período parecen estar registradas en los datos del año 1999. En efecto, cuando dividimos por 11 el número de traducciones aparecidas en 1999 entre cada lengua, el número de traducciones obtenido resulta sumamente cercano al número de traducciones en 1986 y 1987. Los datos obtenidos a partir de esta operación fueron utilizados en nuestras matrices para los años entre 1988 y 1998.

Los datos de México y de Portugal no parecen ser muy confiables. Sin embargo, las lenguas oficiales de estos países son al mismo tiempo las lenguas oficiales de otros países que sí han entregado datos mucho más fiables. Asimismo, las traducciones en España o Brasil son más numerosas, lo que compensa en parte la calidad de los datos de México y Portugal.

Por su parte, como China únicamente ha entregado datos correspondientes al año 1997, los datos de este país fueron suprimidos. Finalmente, destaquemos que antes de 1989 los datos entregados por los "países del este" son los datos oficiales, por lo que las traducciones "prohibidas" no fueron contabilizadas.
} 
90.000 en el 2001. Asimismo, desde 1989 asistimos a una concentración de los flujos de traducciones: no solamente ha aumentado el número de traducciones de manera casi constante, sino que el número de relaciones ha disminuido en un $17 \%$ entre esas dos fechas.

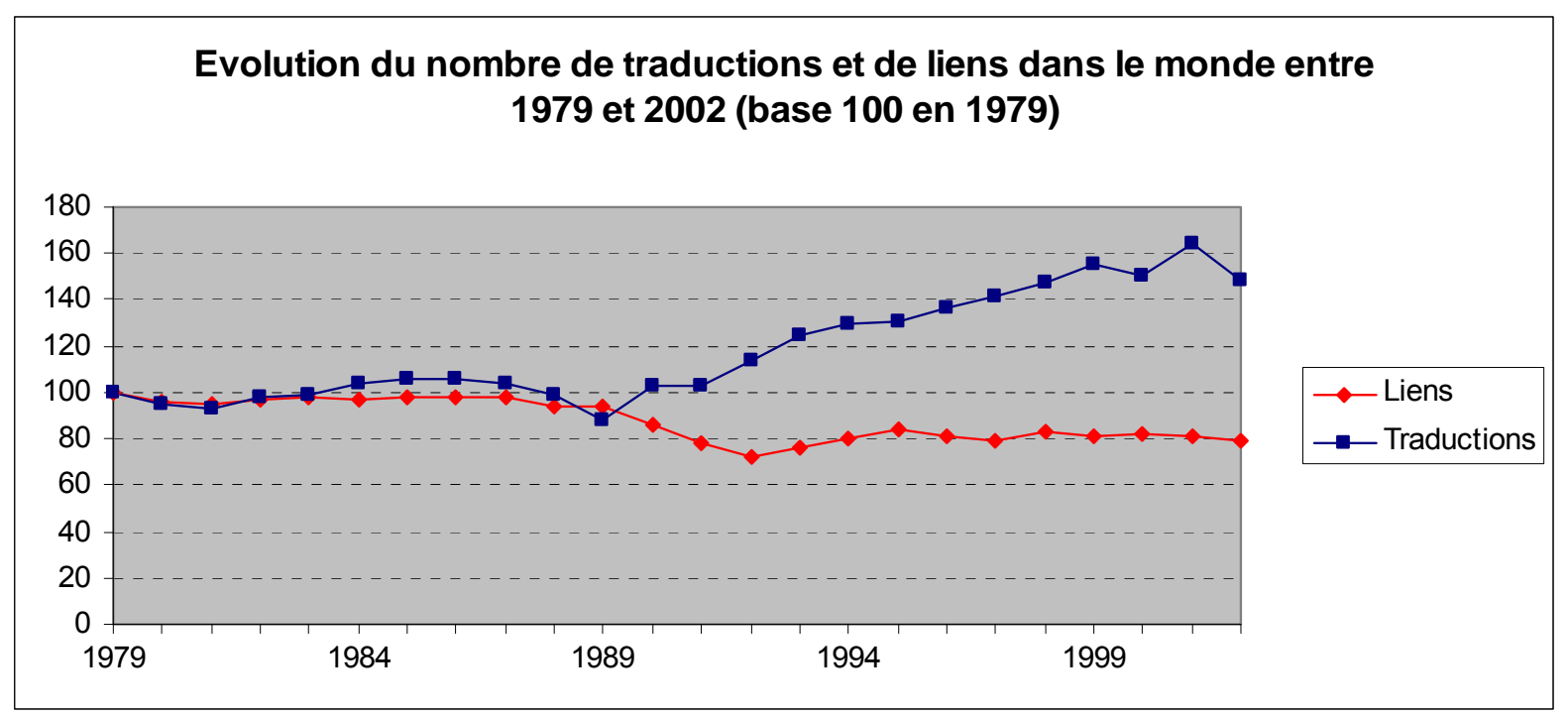

Figura 1. Evolución del número de traducciones (traductions) y de relaciones de traducción (liens) en el mundo entre 1979 y 2002

Esta concentración de flujos de traducciones se contrapone a la definición usual del término "globalización". Mauro Guillen (2001) ha reunido cientos de citas que utilizan el término "globalisation" y que a menudo aportan una nueva definición de este vocablo ${ }^{20}$. Pese a esto, Guillen retiene tres elementos recurrentes: la "compresión" del tiempo y del espacio mundial, la interdependencia y la integración. Al respecto, podemos constatar que en el caso de las traducciones no existe una integración creciente de las lenguas, sino más bien una caída en la densidad de los intercambios.

Entre 1979 y 2002, el número de lenguas traducidas bajó en un $17 \%$ (se cuentan 208 en el 1979 y 172 en el 2002). Esta disminución se concentra en el período 1999-2002. Anteriormente, el número de lenguas traducidas varía de forma muy irregular en torno a 200 (ver Figura 2).

\footnotetext{
20 Guillen, Mauro (2001). Is Globalization Civilizing. Destructive Or Feeble? A Critique of Six Key Debates In The Social Science Literature. Annual Review Of Sociology. 27 (Citado por Hargittai y Centeno (2001). El término globalización, aparecido en 1953, designa en su origen el simple hecho de que un evento adquiera un carácter mundial afectando e interesando al mundo entero. Su sentido ha evolucionado en los años 90 en contacto con el término inglés «globalisation » inventado por Théodore Levitt: la globalización designaría de ahora en adelante "el conjunto de fenómenos, ya sea económicos, culturales o tecnológicos que conducen a una integración creciente de espacios y de hombres a la escala mundial" (Allemand y Ruano - Borbalan, 2005). Para otros, el concepto de "globalización" no sería sino una retórica "invocada por los gobiernos para justificar su sumisión voluntaria a los mercados financieros" (Bourdieu et Wacquant, 2000). Paul Krugman no dice otra cosa cuando escribe que "la retórica de la competitividad es un buen medio para justificar elecciones políticas difíciles o, al contrario, para evitarlas". (Krugman, 2000, Pág. 31).
} 


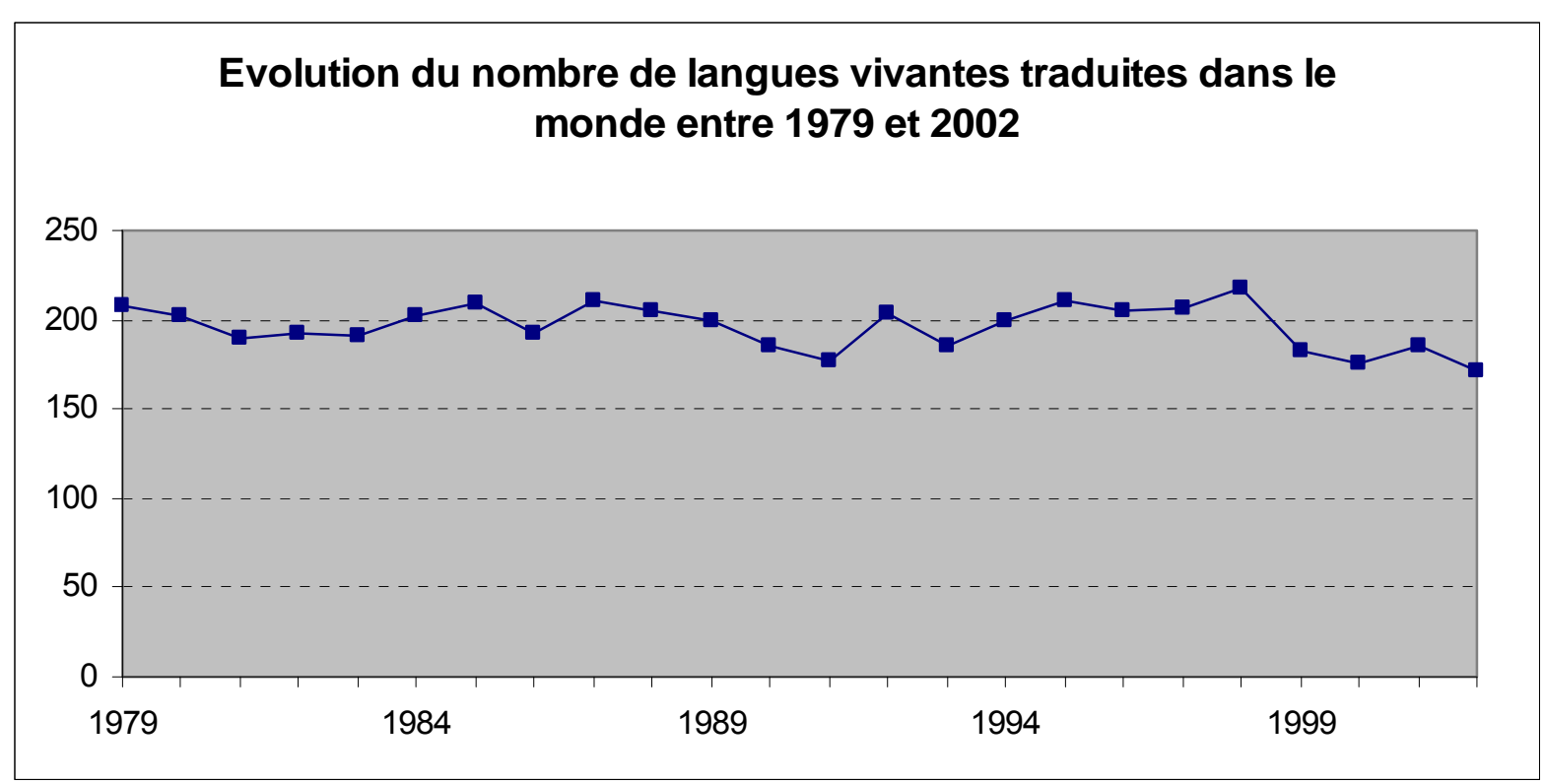

Figura 2: Evolución del número de lenguas vivas traducidas en el mundo entre 1979 y 2002.

Esta brutal caída del número de lenguas traducidas podría ser el signo de un comienzo de monopolio por parte de la lengua inglesa, hipótesis evocada por Mélitz (Mélitz 1998, citado por Heilbron, 1999) ${ }^{21}$. Según un documento de la UNESCO existen aproximadamente 6.000 lenguas en el mundo ${ }^{22}$, pero únicamente un $4 \%$ de ellas son usadas por el $96 \%$ de la población mundial. Más aún, el $50 \%$ de esas 6.000 lenguas estarían en vías de extinción. En Internet, el 90\% de las lenguas del mundo no están representadas...

Siguiendo a Mélitz, podemos formular la siguiente hipótesis: el inglés seria responsable de la caída brutal del número de lenguas traducidas. Dicho de otro modo, las lenguas que desaparecen entre estas dos fechas serían mayoritariamente lenguas traducidas a partir del inglés o en inglés en 1998. Con objeto de probar esta hipótesis, efectuamos una comparación de los datos entre 1998 y 2002. Se contabilizan 217 lenguas traducidas en 1998 y 172 en el 2002, lo que corresponde a una diferencia de 45 lenguas (86 lenguas traducidas en 1998 no lo fueron en el 2002, mientras que 41 lenguas no traducidas en 1998 lo fueron en el 2002).

\footnotetext{
21 « Since the international translation system is so firmly dominated by one hyper-central language, one might presume that translations from other languages will decrease with the consequence of leading to a virtual monopoly for translations from English. In his economic model of the world book market, Jacques Mélitz has explicitly suggested such a possibility: 'If the market in one particular language is sufficiently larger than any other, the total lack of technical barriers to diffusion can lead to the exclusive translation of imaginative works from that particular language into the rest ». Mélitz Jacques (1998). English-Language Dominance, Literature and Welfare. Paris : CREST. Document de Travail $n^{\circ} 9832$.
}

22 Documento disponible en la siguiente dirección: http://portal.unesco.org/culture/fr/ev.phpURL_ID=8270\&URL_DO=DO_PRINTPAGE\&URL_SECTION=201.html 
Cuatro lenguas permiten explicar el $79 \%$ de la disminución constatada: el ruso ( $25 \%)$, el inglés ( $24 \%$ ), el español ( $18 \%$ ) y el francés ( $13 \%$ ). La caída del número de lenguas originales no se debe mayoritariamente al inglés, sino más bien a una concentración de traducciones en Rusia. Si esta concentración es débil en España y Francia (siendo estos dos países grandes traductores, la disminución constatada representa solamente el $10 \%$ de las lenguas traducidas), resulta mucho más fuerte en Rusia. Se destaca en particular la disminución del número de lenguas en la cual el ruso ha sido traducido ("lenguas objetivos"). Mientras que en 1989, se registran 107 lenguas objetivos traducidas en la URSS, la cifra baja a 98 en 1990 y a 72 en 1991. En Rusia, 41 lenguas objetivos fueron traducidas en 1994, 36 en 1995, 21 en 1996 y 4 en 1999. La URSS y luego Rusia han restringido de manera progresiva sus traducciones a los libros traducidos en ruso.

\section{Diferenciar lenguas "originales" de lenguas "objetivos" para explicar la dinámica de flujos de traducciones}

Para explicar la disminución de la diversidad cultural que hemos podido constatar a través de la baja en la densidad de las relaciones de traducción, quisimos estudiar de manera dinámica el reconocimiento de las lenguas. Como veremos, la actualización de esta dinámica permite explicar la disminución de la diversidad cultural.

Diferenciar las lenguas "originales" de las lenguas "objetivos" resulta primordial para evaluar el reconocimiento de una lengua a nivel internacional. En 1979, las traducciones realizadas a partir de obras escritas originalmente en inglés representan el $43 \%$ de las traducciones. Este porcentaje aumenta progresivamente hasta 1998, alcanzando el 48\% . El período 1989-1992 es un período de transición a nivel mundial: también para el inglés, que representa cerca del $60 \%$ de traducciones en 1992 y luego un $62 \%$ en 1996 . Desde esa fecha, el porcentaje del inglés se estabiliza entre 62 y $63 \%$.

Sin embargo, las traducciones en inglés no representan sino el... $5 \%$ de las traducciones en 2002: entre las lenguas "objetivo", el inglés no ha sido nunca mayoritario, alcanzando un máximo de $9 \%$ de las traducciones en 1989. En síntesis, el inglés es fuertemente reconocido a nivel internacional (muchos países traducen a partir de obras escritas en inglés), pero los países anglo-sajones traducen poco en inglés. 


\begin{tabular}{|c|c|c|c|c|c|c|c|c|c|c|c|}
\hline \multicolumn{3}{|c|}{1979} & \multicolumn{3}{|c|}{1989} & \multicolumn{3}{|c|}{1992} & \multicolumn{3}{|c|}{$\underline{2002}$} \\
\hline 1 & Inglés & $43,2 \%$ & 1 & Inglés & $46,6 \%$ & 1 & Inglés & $59,6 \%$ & 1 & Inglés & $62,3 \%$ \\
\hline 2 & Ruso & $12,0 \%$ & 2 & Francés & $11,0 \%$ & 2 & Francés & $12,5 \%$ & 2 & Alemán & $9,3 \%$ \\
\hline 3 & Francés & $11,8 \%$ & . & Ruso & $11,0 \%$ & 3 & Alemán & $8,9 \%$ & 3 & Francés & $9,2 \%$ \\
\hline 4 & Alemán & $9,9 \%$ & 4 & Alemán & $9,2 \%$ & 4 & Italiano & $2,8 \%$ & 4 & Italiano & $3,2 \%$ \\
\hline 5 & Italiano & $2,9 \%$ & 5 & Italiano & $2,9 \%$ & 5 & Ruso & $2,4 \%$ & 5 & Español & $2,9 \%$ \\
\hline 6 & Sueco & $2,3 \%$ & 6 & Español & $2,3 \%$ & 6 & Español & $2,3 \%$ & 6 & Sueco & $1,7 \%$ \\
\hline 7 & Checo & $1,6 \%$ & 7 & Sueco & $2,1 \%$ & 7 & Sueco & $1,7 \%$ & 7 & Ruso & $1,3 \%$ \\
\hline 8 & Español & $1,5 \%$ & 8 & Checo & $1,4 \%$ & 8 & Danés & $1,0 \%$ & 8 & Danés & $0,9 \%$ \\
\hline 9 & Húngaro & $1,5 \%$ & 9 & Húngaro & $1,0 \%$ & 9 & Neerlandés & $0,9 \%$ & 9 & Japonés & $0,9 \%$ \\
\hline 10 & Polaco & $1,2 \%$ & 10 & Danés & $0,9 \%$ & 10 & Árabe & $0,7 \%$ & 10 & Neerlandés & $0,8 \%$ \\
\hline 11 & Danés & $1,1 \%$ & & Neerlandés & $0,9 \%$ & 11 & Checo & $0,7 \%$ & 11 & Portugués & $0,7 \%$ \\
\hline 12 & Neerlandés & $0,9 \%$ & & Polaco & $0,9 \%$ & 12 & Japonés & $0,6 \%$ & 12 & Catalán & $0,6 \%$ \\
\hline 13 & Serbocroata & $0,8 \%$ & 13 & Árabe & $0,8 \%$ & 13 & Polaco & $0,6 \%$ & 13 & Noruego & $0,6 \%$ \\
\hline 14 & Rumano & $0,7 \%$ & & Serbocroata & $0,7 \%$ & 14 & Chino & $0,5 \%$ & 14 & Polaco & $0,5 \%$ \\
\hline \multirow{4}{*}{\multicolumn{2}{|c|}{15 Noruego }} & $0,6 \%$ & & Noruego & $0,6 \%$ & 15 & Hebreo & $0,5 \%$ & 15 & Checo & $0,5 \%$ \\
\hline & & & & Japonés & $0,6 \%$ & 16 & Noruego & $0,5 \%$ & & & \\
\hline & & & & & & 17 & Portugués & $0,5 \%$ & & & \\
\hline & & & & & & 18 & Serbocroata & $0,5 \%$ & & & \\
\hline
\end{tabular}

Tabla 1: Las 15 lenguas más reconocidas en 1979, 1989, 1992 et 2002 (el criterio utilizado es el porcentaje de traducciones realizadas a partir de obras escritas en dicha lengua).

El número de traducciones realizadas a partir de una lengua (clasificada entonces como lengua "original") permite de medir su reconocimiento a nivel internacional. En 1979, tres lenguas representan entre el 10 y el $12 \%$ de las traducciones, mientras que la quinta lengua (el italiano) no cuenta sino con un $3 \%$ de las traducciones.

\section{Evolution de la part des traductions réalisées à partir d'ouvrages écrits en anglais et en russe entre 1979 et 2002 (base 100 en 1979)}

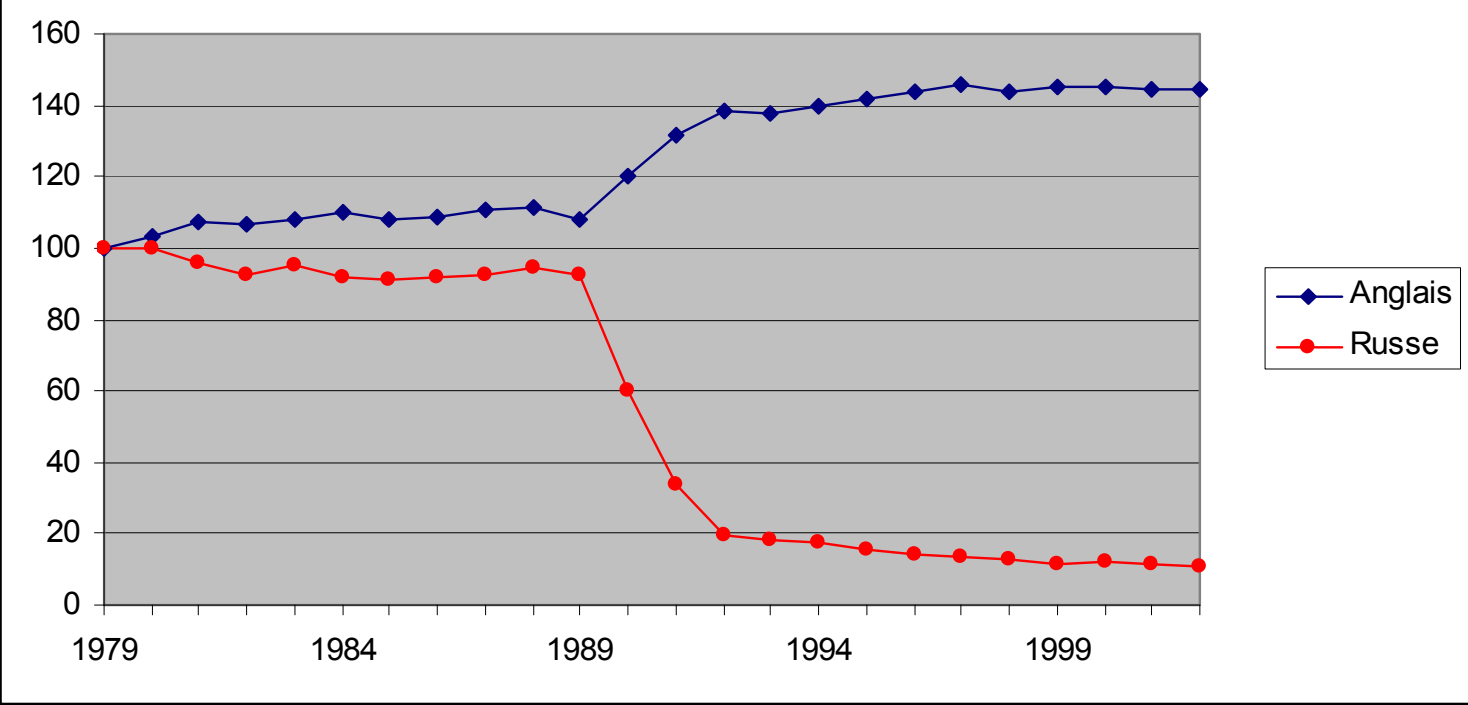

Figura 3. Evolución de la proporción de traducciones realizadas a partir de obras escritas en inglés y en ruso entre 1979 y 2002 (a partir de una base 100 de 1979). 
La Tabla 1 y la Figura 3 muestran que si bien el 12\% de las obras traducidas lo eran a partir del ruso en 1979 (porcentaje que se estabilizó en 1989 al 11\%), el ruso no representa más que el... 2,4\% de las traducciones a nivel mundial en 1992, porcentaje que no hace sino disminuir hasta alcanzar el $1,3 \%$ en el 2002. Si la parte correspondiente al alemán resulta bastante estable, fluctuando entre el 9 y el $10 \%$, la proporción correspondiente al francés, que se elevaba a un $12,5 \%$ de las traducciones a nivel mundial en 1992, pasa a representar un 9,1\% en 1997, estabilizándose en seguida.

Las tres lenguas más reconocidas en el plano internacional representan, entonces, el $81 \%$ de los flujos mundiales de traducciones en 2002 (contra el 67\% en 1979); las siete lenguas más reconocidas el $90 \%$ en 2002 (contra el 84\% en 1979); las quince lenguas más reconocidas el 95\% en el 2002 (contra el 92\% en 1979); etc. Estas cifras muestran claramente que la concentración del flujo de traducciones -ya muy evidente en 1979-, se ve reforzada, sobre todo entre 1989 y 1992.

Para describir los flujos mundiales de traducciones, J ohan Heilbron (1999), inspirándose en los trabajos de Immanuel Wallerstein, utiliza el concepto de "sistema-mundo". En esta estructura de tipo jerárquico, Heilbron distingue una lengua "hiper-central" (el inglés), algunas lenguas centrales (alemán, francés y durante un tiempo el ruso, cada una de ellas representando alrededor del $10 \%$ del "mercado" mundial), las lenguas periféricas (teniendo cada una entre 1 y $3 \%$ del "mercado" mundial) y las lenguas semi-periféricas (menos de un $1 \%$ del "mercado" mundial). Nuestros resultados confirman la hegemonía de la lengua inglesa y su posición en la jerarquía de lenguas a nivel mundial ${ }^{23}$

Dentro de las lenguas "semi-periféricas" o "periféricas", algunas han visto su proporción crecer de manera neta entre 1979 y 2002: el español (de 1,5\% a 2,9\%), el catalán (de $0,1 \%$ a $0,6 \%$ ) y desde 1995 el portugués (de 0,4\% a 0,7\%), así como el japonés (de $0,5 \%$ a $0,9 \%)$. Otras lenguas han conocido una evolución contraria entre 1979 y 2002 : el sueco (de $2,3 \%$ a $1,7 \%$ ), el danés (de $1,1 \%$ a $0,9 \%$ ) y el árabe (de $0,8 \%$ a $0,4 \%$ ).

Sobre todo, podemos destacar que la caída brutal de las traducciones realizadas a partir de obras escritas en ruso coincide con la caída del muro de Berlín. Igualmente, podemos constatar que la proporción correspondiente a la casi totalidad de lenguas de países de la ex URSS cayo fuertemente a partir de 1989. Solo el estonio ha vuelto a alcanzar su nivel de 1979 en el 2002 (0,3\%), luego de una caída al 0,1\% en 1991. Agreguemos a esta constatación las lenguas oficiales de los países de Europa Central: a partir de 1989, la

\footnotetext{
23 El término «periférico » es utilizado de forma diferente por Heilbron, Wallerstein y De Swaan (2001). Por ejemplo, De Swaan distingue cuatro niveles diferentes y considera el árabe o el turco como lenguas « supercentrales », mientras que Heilbron las considera como lenguas periféricas.
} 
parte de las traducciones realizadas a partir de lenguas oficiales de los antiguos países comunistas de Europa Central y del Este (búlgaro, polonés, rumano, eslovaco, checo) cae fuertemente $(5,7 \%$ en 1979 , contra $1,4 \%$ en 2002$)$.

Por otro lado, en el 2002 Serbia y Croacia representan el 0,4\% de las traducciones, mientras que el serbio-croata representaba el 0,5\% en 1992. Es probable que ciertos hechos puedan explicar que el serbio y el croata no hayan conocido una baja de influencia en la escena mundial tan importante como la de las lenguas de las antiguas republicas soviéticas. Recordemos que, luego de su ruptura con Stalin y su exclusión del Kominform en 1948, la Yugoslavia de Tito abandona el modelo soviético de desarrollo para convertirse en un país "no alineado" que se reconocía como comunista al mismo tiempo que afirmaba su independencia en relación a los dos bloques, manteniendo relaciones con países de occidente. En una primera instancia, podemos formular la hipótesis de que la dimensión política es en gran parte responsable del reconocimiento de las lenguas, ya que una "baja" en la calidad de las obras de autores escribiendo en ruso no podría explicar una caída tan brutal, que se desencadena únicamente entre los años $1990-1992^{24}$.

\section{El número de relaciones, nuevo indicador del reconocimiento de las lenguas}

Las lenguas pertenecientes a la periferia del "sistema - mundo" según Heilbron (1999), son numerosas y heterogéneas. Sin embargo, el autor no se aventura a diferenciar entre unas y otras lenguas "periféricas" subrayando, no obstante, que algunas de ellas cuentan con un buen numero de hablantes: Heilbron (1999) destaca que el tamaño de la población que habla una lengua "periférica" (como el chino, el japonés, el árabe o el portugués) y la centralidad de dicha lengua en el sistema internacional no siempre correlacionan ${ }^{25}$.

\footnotetext{
24 De Swaan (2001, pag.10-11) ha subrayado la importancia de esta dimension política: « A map of the present global language system looks quite like a composite of political maps from the eighteenth, nineteenth and twentieth centuries. It shows how much language constellations are determined by political events, but also how they often survive long after this political base has disappeared. Thus, Spanish and Portuguese came to the southern part of the western hemisphere as colonial languages, and so did English and French in North America. And although almost the entire continent became independent of the European mother countries between the end of the eighteenth and the middle of the nineteenth century, the languages of the former colonizers, English, Spanish and Portuguese, still prevail there ».

Entre los capítulos 4 y 7 de esta obra, De Swaan explica el mantenimiento de las lenguas de los antiguos países colonizadores. Mediante numerosos ejemplos, el autor muestra como el dominio de la lengua del antiguo país colonizador es importante para acceder a las esferas del poder en los antiguos países colonizados. Únicamente Indonesia constituye una excepción por diversas razones (entre otras, el movimiento nacionalista y la débil difusión del holandés, tanto sobre este territorio como a nivel internacional). Para más detalles del caso de Indonesia, ver el capítulo 5 de esta obra.

25 Heilbron (1999): « Among these peripheral languages there are Chinese, Japanese, Arabic, and Portuguese, each representing a very large number of speakers, yet occupying a peripheral position in the translation system. The size of language groups is clearly not decisive for their degree of centrality (subrayo) in the translation system ».
} 
Ahora bien, nos parece que la definición misma del indicador de centralidad de una lengua plantea ciertos problemas. Como lo dijimos anteriormente, la fiabilidad de los datos del Index Translationum resulta incierta, particularmente a causa de la falta de consenso sobre la definición de libro. Del mismo modo, consideramos que el número de traducciones no constituye un indicador suficiente para evaluar el reconocimiento de lenguas a nivel mundial (a excepción del inglés, el francés, el alemán y el ruso antes de 1989 y de las tres lenguas citadas después de 1992) por tres razones: la calidad de los datos, las variaciones en las definiciones de libro y la débil representación de las lenguas periféricas que siguen este criterio. En efecto, aparte del italiano, el español, el sueco y el ruso, una lengua periférica no representa más de un $0,9 \%$ de las traducciones en el 2002 (ver más arriba). En consecuencia, resulta difícil distinguir con claridad las lenguas periféricas de mayor reconocimiento.

Tomemos un ejemplo: en el 2002, el número de traducciones realizadas a partir de obras escritas en catalán es ocho veces mayor que las hechas en esloveno. Sin embargo, el número de lenguas para las cuales se realizó una traducción desde una obra escrita en catalán es prácticamente el mismo que en esloveno (17 versus 15). Esta diferencia se puede explicar por el hecho de que el catalán se traduce mayoritariamente en un país que traduce mucho (España), mientras que la Eslovenia traduce muchas menos obras. Considerando esto: ¿podemos afirmar que el catalán es más reconocido que el esloveno en el plano internacional?

El método de análisis de redes sociales ofrece nuevas herramientas para evaluar el reconocimiento internacional de las lenguas. Dicho método permite calcular el número de relaciones de traducción para cada lengua. En análisis de redes, el número total de relaciones de un actor es Ilamado Grado de Centralidad. Este indicador puede calcularse para los dos sentidos de la traducción: los dos índices obtenidos se denominan semigrado interior y semi-grado exterior. El ejemplo del inglés demuestra que resulta particularmente útil distinguir estos dos índices en nuestro estudio. Aquí utilizaremos el término de semi-grado interior para calificar las elecciones "recibidas" por una lengua $X$. Dicho de otra forma: el número de lenguas en las cuales ha sido traducida una obra escrita originalmente en la lengua $X$. 


\begin{tabular}{|c|c|c|c|c|c|c|c|c|c|c|c|}
\hline \multicolumn{3}{|c|}{1979} & \multicolumn{3}{|c|}{1989} & \multicolumn{3}{|c|}{1992} & \multicolumn{3}{|c|}{2002} \\
\hline 1 & Ruso & 107 & 1 & Ruso & 109 & 1 & Inglés & 96 & 1 & Inglés & 72 \\
\hline 2 & Inglés & 85 & 2 & Inglés & 96 & 2 & Ruso & 59 & 2 & Francés & 64 \\
\hline 3 & Alemán & 67 & 3 & Francés & 73 & 3 & Francés & 54 & 3 & Alemán & 56 \\
\hline 4 & Francés & 65 & 4 & Alemán & 63 & 4 & Alemán & 47 & 4 & Español & 49 \\
\hline 5 & Italiano & 43 & 5 & Español & 43 & 5 & Español & 40 & 5 & Ruso & 44 \\
\hline . & Checo & 43 & 6 & Italiano & 41 & 6 & Sueco & 36 & 6 & Danés & 42 \\
\hline 7 & Español & 40 & 7 & Sueco & 37 & 7 & Italiano & 35 & . & Italiano & 42 \\
\hline 8 & Polaco & 38 & 8 & Polaco & 36 & 8 & Danés & 33 & 8 & Sueco & 40 \\
\hline 9 & Sueco & 37 & . & Checo & 36 & 9 & Árabe & 32 & 9 & Árabe & 36 \\
\hline 10 & Húngaro & 35 & 10 & Japonés & 35 & 10 & Japonés & 30 & . & Portugués & 36 \\
\hline 11 & Danés & 34 & 11 & Árabe & 34 & 11 & Chino & 29 & 11 & Japonés & 34 \\
\hline 12 & Búlgaro & 33 & 12 & Danés & 33 & . & Neerlandés & 29 & 12 & Neerlandés & 33 \\
\hline . & Japonés & 33 & 13 & Noruego & 30 & 13 & Finés & 27 & . & Noruego & 33 \\
\hline 14 & Portugués & 30 & . & Ucraniano & 30 & . & Checo & 27 & . & Checo & 33 \\
\hline 15 & Árabe & 29 & 15 & Lituano & 29 & 15 & Hebreo & 26 & 15 & Hebreo & 32 \\
\hline . & Serbocroata & 29 & & Serbocroata & 29 & 16 & Noruego & 25 & 16 & Polaco & 31 \\
\hline 17 & Ucraniano & 28 & 17 & Portugués & 27 & . & Polaco & 25 & 17 & Húngaro & 28 \\
\hline 18 & Neerlandés & 27 & 18 & Neerlandés & 26 & . & Portugués & 25 & 18 & Chino & 26 \\
\hline . & Persa & 27 & & Húngaro & 26 & 19 & Húngaro & 24 & . & Finés & 26 \\
\hline & Noruego & 26 & 20 & Chino & 25 & . & Serbocroata & 24 & 20 & Griego & 25 \\
\hline & & & & Rumano & 25 & & & & . & Persa & 25 \\
\hline
\end{tabular}

Tabla 2. Las 20 lenguas más reconocidas en 1979, 1989, 1992 y 2002 según el criterio de semi-grado interior

En el 2002, los semi-grados interiores del catalán y el esloveno son bastante equivalentes (17 para el catalán, 15 para el esloveno) al contrario del número de traducciones (las cifras fueron citadas anteriormente). Si el catalán era la duodécima lengua más traducida en el 2002, utilizando como criterio el número de traducciones, esta lengua no alcanza sino la vigésimo sexta posición al utilizar el criterio de semi-grado interior. Al contrario, el árabe, cuyo número de traducciones es inferior al del catalán en el 2002, tiene un semi-grado interior más de dos veces superior (36 contra 17).

Estos ejemplos muestran que el criterio de semi-grado interior es complementario, e incluso en ocasiones preferible, al del número de traducciones en el estudio de las lenguas Ilamadas "periféricas". El reconocimiento de una lengua no puede ser medido únicamente en relación al número de traducciones, debido especialmente a la falta de armonización internacional en la definición de libro, lo que podría llevar a subestimar el 
número de traducciones de ciertos países.

Destaquemos, por otro lado, que el ruso es según este criterio la lengua más reconocida hasta 1989. Ahora bien, anteriormente hemos observado la hegemonía de la lengua inglesa, incluso antes de 1989, lo que nos lleva a considerar las insuficiencias del criterio de semi-grado interior.

Así podemos deducir que, si el número de relaciones da una estimación del reconocimiento de una lengua, conocer los "miembros" de las relaciones y sus posiciones respectivas en el "sistema-mundo" resulta igualmente importante. De esta forma, a continuación intentaremos describir y comprender la dinámica de este "sistema-mundo" entre 1979 y 2002.

\section{EI "sistema-mundo" entre 1979 y 2002}

\section{Las relaciones "preferenciales" más frecuentes}

Para comprender el "sistema-mundo", es necesario conocer las lenguas que más participan en el reconocimiento de cada una de las otras lenguas. Para responder al problema planteado, nos vimos enfrentados a una densidad demasiado importante al utilizar las matrices cuya construcción se describe más arriba ${ }^{26}$. Esto se explica por nuestra opción de considerar que existe una relación entre dos lenguas si al menos una traducción ha sido realizada. Por ejemplo, se contabilizan en 1979 un promedio de 5.000 traducciones del alemán al inglés, lo que constituye una relación entre el alemán y el inglés, pero también se cuenta una veintena de traducciones del catalán al inglés, lo que igualmente constituye una relación entre el catalán y el inglés.

Para reducir esta densidad demasiado elevada, elegimos el siguiente método. En una primera instancia, conservamos solamente las 139 lenguas más traducidas en el curso de los 24 años estudiados (la suma de las traducciones de estas 139 lenguas representa el... 99\% del conjunto de traducciones). Precisemos que sobre los 24 años del estudio, se contabilizan solamente 6 traducciones entre las lenguas menos traducidas, lo que justificaría la utilización del término "periférico" a ese conjunto de lenguas.

Enseguida, utilizamos el criterio de independencia estadística. Más precisamente, comparamos el número de traducciones de matrices con aquel que habría existido si no existieran preferencias en el flujo de traducciones. Por ejemplo, en 1979 se contabilizan 1.400 traducciones en español a partir de obras escritas en francés. Ahora bien, si no

\footnotetext{
${ }^{26}$ Así se calcula la densidad: el número de relaciones observadas dividido por el número de relaciones posibles.
} 
existieran preferencias, habríamos obtenido un total de 773 traducciones ${ }^{27}$. El número observado es entonces 1,8 veces más importante que en el caso de la independencia estadística. De esta manera podemos concluir que el español participa a un mayor reconocimiento mundial del francés (ya que 1,8 es mayor que 1), existiendo una relación preferencial entre esas dos lenguas en ese sentido de traducción en el año 1979. Esta comparación fue efectuada en las 24 matrices compuestas con las 139 lenguas más traducidas.

Con el fin de simplificar aún más el análisis, hemos buscado las relaciones preferenciales más frecuentes: solo las relaciones preferenciales que perduraron sobre un período de cinco años fueron conservadas en cuatro matrices, cada una representando un período de cinco años (1980-1984, 1985-1989, 1992-1996, 1997-2001) ${ }^{28}$. Pese a estas simplificaciones, las descripciones que se muestran a continuación pueden parecer bastante pesadas de seguir. Sin embargo éstas resultan necesarias, ya que hasta hoy el "sistema-mundo" nunca ha sido objeto de una descripción de este tipo.

\section{Descripción del “sistema-mundo" durante el período 1979-1984}

\section{Leyenda $^{29}$ :}

- El tamaño de los puntos es proporcional al número de relaciones preferenciales de la lengua, esto es, a la suma de relaciones preferenciales "recibidas" o "emitidas". Por ejemplo, el italiano y el francés tienen una relación preferencial en los dos sentidos de la traducción. El francés y el italiano tienen, entonces, dos relaciones preferenciales.

- Los conjuntos de lenguas que son fuertemente conectados entre si, así como las lenguas que tienen características comunes aparecen con el mismo color (ver los comentarios para más precisiones)

a) El inglés y el ruso son las dos lenguas más centrales en el período 1980-1984. La posición entre los dos bloques es clara: el inglés tiene relaciones preferenciales de traducción con todas las lenguas oficiales de los países occidentales y el ruso presenta lazos preferenciales de traducciones con todas las lenguas oficiales de países del bloque comunista. A diferencia del ruso que se conecta únicamente con el finés, el inglés si se

\footnotetext{
27 En 1979 se cuentan 6.516 traducciones en español, 6.706 traducciones a partir del francés y 56.556 traducciones en total. En el presente caso, deberíamos haber observado $[(6.516 \times 6.706) / 56.556]=772,6$ traducciones.

${ }^{28}$ Los años 1990 y 1991 son años de “transición”, por esta razón no los hemos incluido. Con el fin de comparar cuatro períodos de una misma duración (cinco años), los años 1979 y 2002 fueron igualmente excluidos.

29 Esta leyenda es la misma para los tres gráficos siguientes.
} 
conecta con diversos miembros del otro bloque (estoniano, ucraniano, armenio, polonés, húngaro, rumano).

b) El alemán y el francés son las otras dos lenguas importantes: ellas se conectan fuertemente con las lenguas oficiales de los países vecinos. El alemán se conecta, por ejemplo, con el francés, el holandés, el polonés, el húngaro y el esloveno (cabe recordar que Hungría y el territorio actual de Eslovenia son limítrofes con Austria). El francés se relaciona fuertemente con lenguas de países mediterráneos (portugués, español, catalán, italiano, griego), aún cuando también existen lazos con lenguas situadas más al norte de Europa (holandés, alemán). 


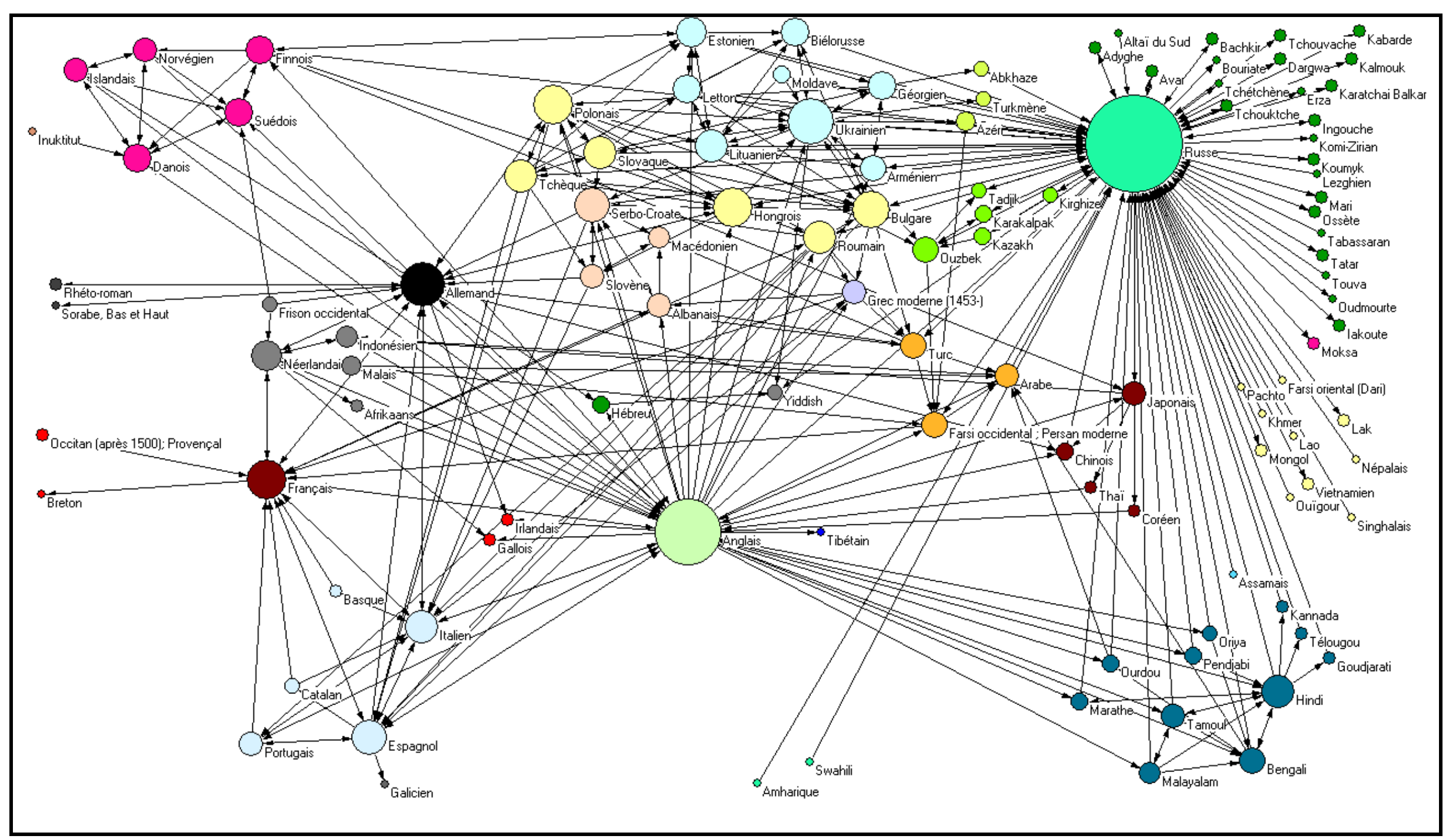

Figura 4. Las relaciones preferenciales de traducción durante el período 1980-1984. 
c) En la "periferia", encontramos varios grupos de lenguas.

* Grupos que comprenden una gran mayoría de lenguas de países occidentales

En primer lugar, se puede destacar que las lenguas del norte de Europa (el islandés, el noruego, el sueco, el finés y el danés) se conectan fuertemente entre ellas. Todas estas lenguas tienen una relación preferencial con el inglés, pero muy pocas con otras lenguas, con la excepción de algunos lazos preferenciales con lenguas germánicas (alemán, frisón "occidental") y los lazos exteriores del finés (con el estoniano y el ruso, en particular). Estas son las lenguas oficiales de los estados miembros del Consejo Nórdico, fundado en 1952.

Se observa igualmente un bloque de lenguas reunidas en torno al holandés: el frisón "occidental" - lengua hablada corrientemente en el norte de Holanda-, pero también tres lenguas de antiguos países colonizados por Holanda (afrikáans, indonesio, malayo).

A continuación, se destaca la presencia de un grupo de lenguas del cual hacen parte el español, el portugués y el italiano. En este conjunto figuran igualmente lenguas como el vasco y el catalán. Este bloque se ve particularmente conectado con el inglés, el francés, el rumano, así como con el alemán y el griego. El italiano tiene también relaciones preferenciales con dos lenguas habladas especialmente en el país que en su época se llamaba Yugoslavia (el serbio-croata y el esloveno).

* Un grupo compuesto en una gran mayoría por lenguas oficiales de países comunistas

El serbio-croata, el esloveno, el macedonio, el polonés, el eslovaco, el húngaro, el búlgaro, el rumano, el albanés y el griego son parte de este conjunto. Las lenguas oficiales de los países limítrofes están casi todas ligadas de manera preferencial entre ellas: en este caso el criterio geográfico resulta claramente decisivo. Así, el polonés se ve ligado al checo y al eslovaco, el macedonio al albanés y el griego al albanés o al búlgaro. Solo las relaciones búlgaro/serbio-croata y griego/macedonio son la excepción. Las relaciones preferenciales de traducción al interior de este conjunto sobrepasan los desacuerdos políticos entre países comunistas "ligados" a Moscù, especialmente a través del pacto de Varsovia (Polonia, Checoslovaquia, Hungría, Bulgaria y Rumania, en amarillo en la Figura 4), los países comunistas pero bastante independientes de Moscú (Yugoslavia - ver anteriormente- y Albania, país que dejo el pacto de Varsovia en 1968) y los países no comunistas (Grecia).

No obstante, se pueden percibir estos diferendos al observar las relaciones preferenciales de traducción hacia el exterior de este conjunto. Todas las lenguas de los países miembros del Pacto de Varsovia (polonés, checo, eslovaco, húngaro, búlgaro, rumano) 
tienen una relación preferencial con el ruso y con lenguas de antiguas repúblicas soviéticas, con excepción del rumano. Al contrario, las lenguas de los tres países más independientes de la URSS (serbio-croata, esloveno, macedonio, albanés, griego) no tienen lazos preferenciales con las ex-repúblicas soviéticas salvo el serbio-croata, que se ve conectado con el ucraniano y el ruso. El criterio decisivo para explicar las relaciones preferenciales de traducción en este caso es el criterio político, que sin embargo no resulta independiente del criterio geográfico (es mas fácil la independencia política de un país respecto a otro cuando está alejado geográficamente, ceteris paribus)

* Tres grupos de lenguas de repúblicas soviéticas

Acabamos de evocar la existencia de relaciones entre las lenguas de países comunistas unidos a la URSS por el Pacto de Varsovia y ciertas lenguas de antiguas repúblicas soviéticas. Estas lenguas son el estonio, el letón, el lituano, el bielorruso, el ucraniano, el moldavo y el georgiano. A éstas podemos agregar el armenio, conectado con el ucraniano, el lituano y el georgiano. Estas lenguas se observan fuertemente relacionadas entre ellas y en particular en torno al ucraniano, que se conecta con seis de las siete lenguas de este grupo, pero también (y es una tautología) con el ruso.

El segundo grupo es constituido por el azerí, el abjasio (lengua de la república de Georgia) y el turcomano. Estas lenguas no se relacionan entre ellas, pero tienen la particularidad de conectarse con una de las 8 lenguas citadas precedentemente, además del ruso. Se trata del ucraniano para el azerí y el turcomano, así como el georgiano para al abjasio.

El tercer grupo se compone de cinco lenguas reunidas en torno al uzbeko, que se conecta con el ucraniano. Las otras cuatro lenguas son el kazajo, el karakalpaco (hablado en el norte de Uzbekistán) el kirguiz y el tayiko. Éstas son habladas en las repúblicas soviéticas del continente asiático, como las lenguas del grupo precedente. Estos dos últimos grupos se ven bastante aislados del primero, en especial porque los países donde se hablan estas lenguas están alejados geográficamente de las antiguas repúblicas soviéticas situadas en Europa.

* Un grupo de lenguas habladas en Rusia

El ruso tiene relaciones preferenciales y exclusivas de traducción con una veintena de lenguas habladas en la antigua URSS, que no se conectan entre ellas. Se puede citar como ejemplo el tártaro.

* Tres grupos de lenguas asiáticas

El primero de estos conjuntos está compuesto por diez lenguas, entre las cuales se 
encuentran el lao y el vietnamita, que se relacionan exclusivamente con el ruso.

El segundo grupo está compuesto por cuatro lenguas relacionadas con el inglés. Se trata del chino, el tailandés y el coreano, que a su vez se relacionan con el japonés. El chino y el japonés tienen relaciones preferenciales de traducción con el ruso, el japonés con el finés (algo que resulta muy puntual) y el indonesio, junto al chino, con el alemán.

El tercer grupo se compone de una decena de lenguas indias que tienen la particularidad - ocho de entre ellas-, de tener relaciones preferenciales de traducción casi exclusivas con el inglés y el ruso (las excepciones son el urdu y el bengalí que tienen un lazo preferencial con el árabe). Todas estas lenguas se conectan al bengalí y/o al hindi. De Swann (2001) consagra un capítulo entero a estas lenguas que se hablan únicamente en la India ${ }^{30}$. En ese país se cuentan en promedio 800 lenguas, pero solamente 18 tienen carácter oficial ${ }^{31}$, entre las cuales se encuentran la decena de lenguas de nuestro grupo. Al interior de éste, existen ciertas divisiones lingüísticas entre las lenguas del norte y del sur del sub-continente indio ${ }^{32}$, divisiones que se encuentran de un momento a otro en nuestros datos. Por ejemplo el telugú, que es la tercera lengua en término de hablantes, no tiene sino una única relación con el hindi (primera lengua más hablada) durante los cuatro períodos considerados y ninguna relación con el bengalí (segunda lengua más hablada).

\section{* Un último grupo de tres lenguas}

Este grupo reúne al turco, al persa y al árabe. Si bien estas tres lenguas tienen lazos preferenciales entre ellas y con el ruso, sus otras relaciones son muy diversas. El turco se relaciona fuertemente con lenguas de países europeos vecinos (albanés, macedonio,

\footnotetext{
30 «The Indian language constellation comprises more than India alone; it also covers Pakistan, Bangladesh, Nepal, Bhutan and Sri Lanka, and other countries (De Swaan, 2001, Pág.61) ».

31 «Some eight hundred languages are spoken in India, and the most recent census lists more than 1600 names for them. However, only eighteen are listed in the Eighth Schedule attached to the constitution. With some exceptions, each one of these is the official language in one or more of the constituent states of the Union. According to the 1991 census, more than 96 per cent of all Indians speak one of these scheduled languages as their mother tongue. Six languages, Bengali, Hindi, Marathi, Tamil, Telugu and Urdu, together are spoken by three-quarters of the population as their mother tongue. Hindi is by far the largest, spoken by 337 million people, or 40 per cent of the population, in one variety or another. Bengali, with more than 8 per cent, comes second. Telugu, a Dravidian language, takes third place with almost 8 per cent (De Swaan, 2001, Pág.61)».

«Originally the Vita Schedule contained fourteen languages: Assamese, Bengali, Gujarati, Hindi, Kannada, Kashmiri, Marathi, Malayalam, Oriya, Punjabi, Sanskrit, Telugu, Tamil, and Urdu. Sindhi was added later. In 1992, with the seventy-first amendment to the constitution, Konkani, Manipuri and Nepali were added to the list. » (Cf. Manorama Yearbook, 1996, Pág.464, citado por De Swaan, 2001, nota 2 del capítulo 4, Pág.202).

32 «In a linguistic perspective, the main division is between the Indo-European, "Indo-Aryan" Ianguage family of the north and Dravidian languages of the southern part of the subcontinent. And it is this distinction that also marks a sharp divide of mutual unintelligibility (De Swaan, 2001, Pág.61-62) ».

« The most important [Dravidian languages] being Telugu, Tamil, Kannada and Malayalam, comprising together some 22 per cent of all Indian mother tongue speakers » (De Swaan, 2001, nota 5 del capítulo 4, Pág.203).
} 
griego, búlgaro, romano), así como con el alemán, lo que resulta comprensible dada la población turca o de origen turco viviendo en Alemania. El persa se conecta con lenguas de países limítrofes (Azerbaiyán) o vecinos (Uzbekistán) de Irán y el árabe con el urdu o el bengalí. El malayo y el indonesio mantienen relaciones de traducción preferenciales con el persa y/o el árabe.

\section{* Las otras lenguas}

Primero están aquellas lenguas conectadas exclusivamente con el idioma (a menudo oficial) del país donde se hablan. Es el caso del bretón y el occitano en Francia, del gallego en España o del inuit en Groenlandia (territorio danés). Luego se observan casos como el de dos lenguas africanas, ligadas exclusivamente al ruso (el amárico y el swahili), o bien dos lenguas que se relacionan preferentemente con el inglés por razones evidentes (el irlandés y el galo), y del tibetano que tiene un lazo preferencial exclusivo con el inglés. Finalmente, el hebreo y el yiddish, dos lenguas habladas principalmente en las comunidades judías, tienen relaciones de traducciones preferenciales entre ellas. Sin embargo, el hebreo se conecta igualmente al inglés y al alemán, mientras que el yiddish se relaciona con el ucraniano y el ruso. Estas relaciones reflejan los movimientos migratorios de las poblaciones que hoy hablan estas dos lenguas.

Considerando lo anterior, podemos hacer un primer balance. Dejando de lado el inglés, son sobre todo las lenguas de países vecinos las que participan en el reconocimiento de los idiomas. La dimensión política resulta igualmente importante: los antiguos países de la URSS tienen relaciones preferenciales de traducción entre ellos. Otro ejemplo: las lenguas indias no poseen lazos preferenciales de traducción con las lenguas de la ex URSS (aparte del ruso) ni con las lenguas de otros países asiáticos, pese a su proximidad geográfica. Además, las especificidades coloniales permanecen. Por ejemplo, el holandés mantiene relaciones preferenciales con el afrikáans, el indonesio y el malayo, lenguas de antiguos países colonizados por Holanda, etc.

\section{Las evoluciones del "sistema-mundo" entre 1979 y 2002}

Podemos constatar una importante estabilidad de las relaciones preferenciales de traducción entre 1985-1989 y 1980-1984, aún cuando los datos utilizados son bastante diferentes entre los períodos considerados. 


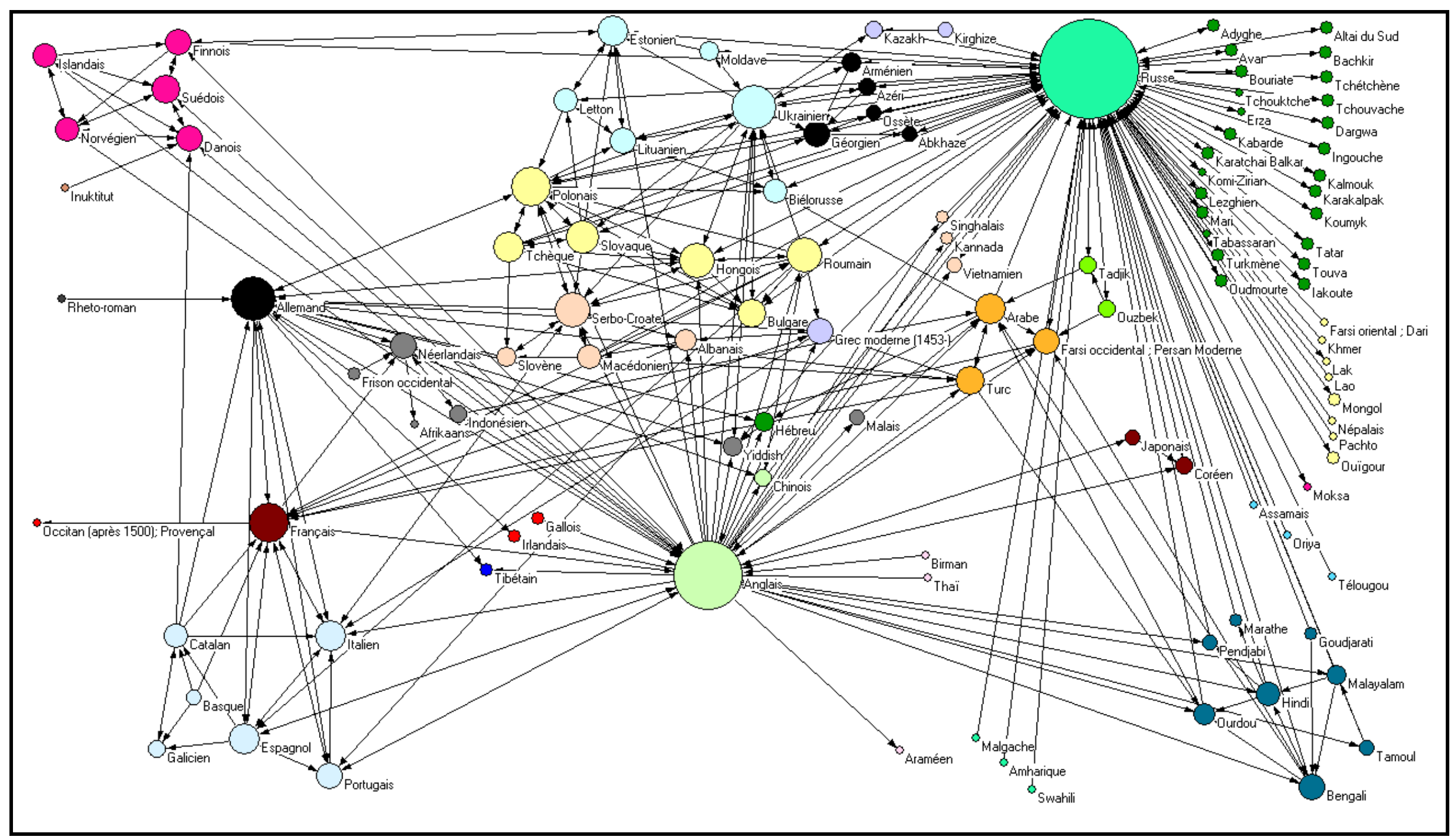

Figura 5. Las relaciones preferenciales de traducción durante el período 1985-1989. 
Sin entrar en mayores detalles, se observa

en particular la desaparición de ocho relaciones preferenciales entre las ocho lenguas de ex repúblicas soviéticas más conectadas con los países miembros del Pacto de Varsovia ${ }^{33}$. Algo similar sucede con el grupo de lenguas de la India. Además, el grupo de cinco lenguas reunidas en torno al uzbeko se desintegra: el tayiko y el uzbeko se observan aislados de las lenguas de las republicas soviética con excepción - evidentemente- del ruso.

Por otra parte, el turco pierde las relaciones preferenciales de traducción con cuatro lenguas de países mediterráneos. Finalmente, se observan los cambios de relaciones preferenciales de ciertas lenguas asiáticas como el chino o el tailandés que dejan de conectarse con el japonés (lo que es provisorio), o el malayo, que no continua teniendo lazos preferenciales con el holandés. Las otras evoluciones son más bien menores.

Después de 1989 se observa una "gran transformación". Muchos de los grupos fácilmente identificables antes de 1990 se rompen.

En efecto, si el ruso mantiene relaciones de traducciones preferenciales con numerosas lenguas de países de la ex URSS, estos últimos ya casi no presentan relaciones de traducciones preferenciales entre ellos, a excepción del letón y el estonio. Más aún, se observa la ausencia de relaciones entre las lenguas de las antiguas repúblicas soviéticas (entre ellas el ruso) y las lenguas de países del Este en el período 1992-1996. Por ultimo, constatamos que el número de lenguas del territorio ruso traducidas de manera preferencial disminuye fuertemente (de una veintena a ocho).

\footnotetext{
${ }^{33}$ Estas lenguas son el armenio, el bielorruso, el estonio, el georgiano, el letón, el lituano, el moldavo y el ucraniano. Al respecto, hay que recordar que la información proveniente de los países del bloque comunistas son datos oficiales, en los que se excluye la literatura prohibida.
} 


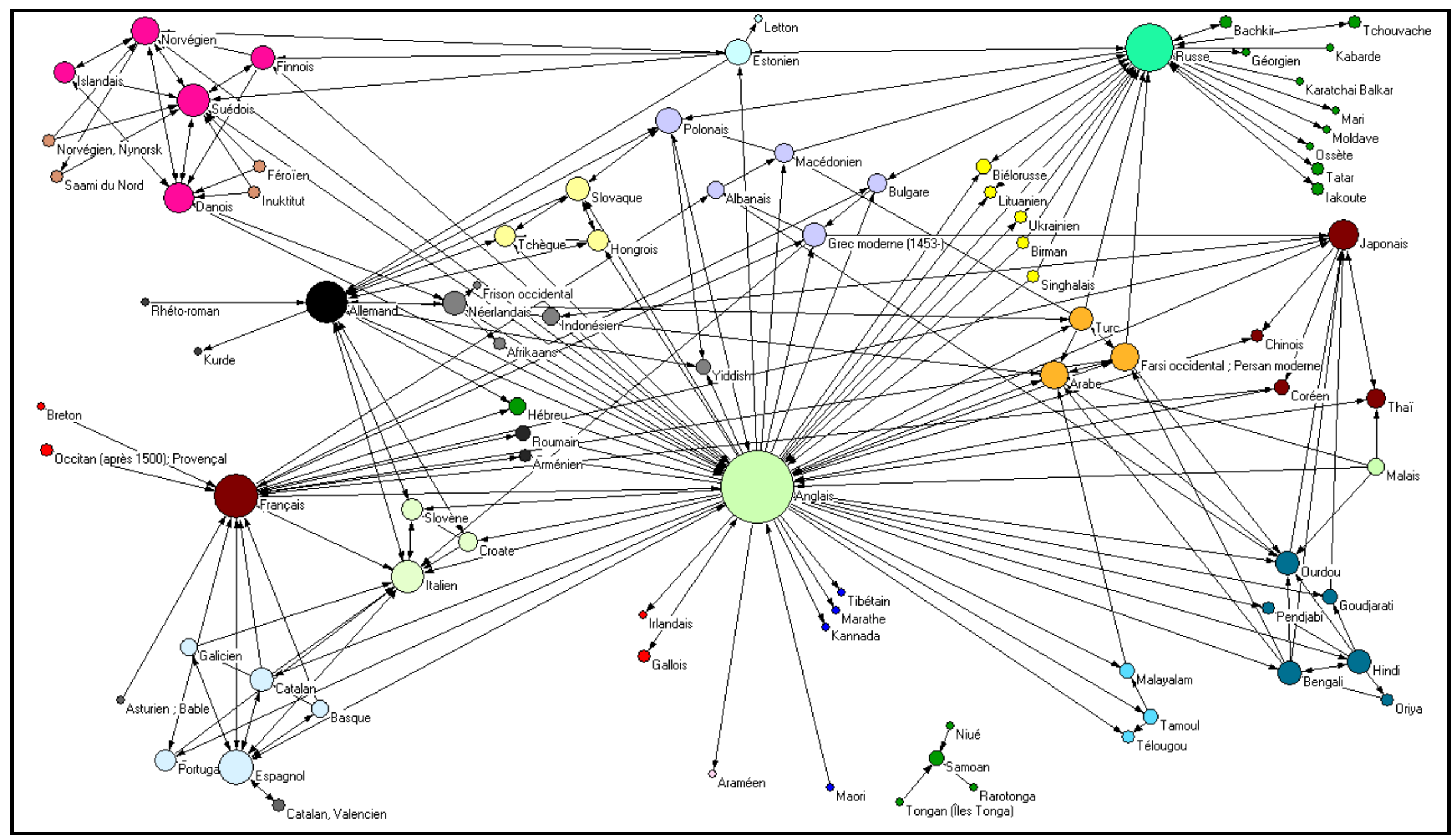

Figura 6. Las relaciones preferenciales de traducción durante el período 1992-1996. 
Volvemos encontrar aquí la explicación de la baja del número de relaciones de traducción observada después de 1989. La concentración del flujo de traducciones a nivel mundial durante este período se explica por la caída de la URSS: los lazos preferenciales de traducciones entre las republicas soviéticas, garantizados por la URSS se atenúan progresivamente. En efecto, a partir de 1991, las antiguas repúblicas soviéticas acceden progresivamente a la independencia. Poco a poco, los datos del Index Translationum aparecen en la parte europea de la ex URSS (1990 Estonia, 1991 Bielorrusia, 1993 Lituania y Moldavia, 1994 Letonia, 1995 Ucrania, 2001 Azerbaiyán), mientras que las otras antiguas republicas soviéticas no entregan aún datos. Como lo destacáramos anteriormente, las lenguas habladas en los países de la parte asiática de la URSS (azerí, kazajo, kirguiz y uzbeko, en particular) ya estaban fuertemente aisladas de las otras lenguas habladas en las republicas soviéticas antes de 1989, con excepción del ruso y del ucraniano en el caso del kazajo o el kirguiz.

La caída de la URSS no solo tuvo repercusiones negativas para los lazos preferenciales de traducciones entre las lenguas de las repúblicas soviéticas, sino también en el reconocimiento mundial del ruso (la casi totalidad de las lenguas de los países de Asia entre las que se encuentran las lenguas indias- no presentan más relaciones preferenciales de traducción con el ruso después de 1989) y sobre las relaciones preferenciales de traducción entre los antiguos miembros del Pacto de Varsovia. Así, el rumano y el búlgaro dejan de tener relaciones preferenciales con el checo, el eslovaco, el polonés y el húngaro.

Agreguemos a esto el desmembramiento de las relaciones entre las lenguas de la ex Yugoslavia, que se dividen en varios grupos: el croata y el eslovaco forman un sub-grupo cohesivo con el "vecino" italiano y tienen las mismas relaciones (a excepción de las relaciones preferenciales con el francés, que en un primer tiempo son una exclusividad del italiano). El macedonio tiene otras relaciones preferenciales de traducción y el serbio no está más presente, por razones evidentes.

En consecuencia, se observa una disminución espectacular de la influencia del ruso a partir de la caída del muro de Berlín y una centralidad aún más importante del inglés en la red de lazos preferenciales de traducción. A modo de ejemplo, el lituano, el checo, el eslovaco, el esloveno y el búlgaro pasan a tener relaciones preferenciales de traducción con el inglés. Estas evoluciones se reflejan en la proporción de traducciones realizadas a partir de obras escritas en ruso (que cae del $11,1 \%$ al 2,4\% entre 1989 y 1992) y del inglés (cuya proporción aumenta del $46,6 \%$ al 59,9\% entre esas mismas dos fechas, ver 
más arriba) ${ }^{34}$.

Subrayemos igualmente la extensión del conjunto de lenguas de países nórdicos compuesto hasta 1989 por el islandés, el noruego, el sueco, el finés, el danés y el inuit hablado en Groenlandia. A éstos hay que agregar, a partir de 1992 el kalaallisut (o groenlandés), el feroés (de las Islas Feroe, territorio danés), el sami (hablado en Noruega, Suecia y Finlandia, y el nynorsk ${ }^{35}$. Destaquemos también la desaparición definitiva de las relaciones del alemán con el cataban, el español y el portugués. Por último, la aparición de nuevos datos nos hace descubrir un nuevo grupo compuesto por lenguas de las islas de Oceanía (el samoano y el tongano, por ejemplo). Estas se ven aisladas de otras lenguas.

En relación al período precedente, se destaca un aumento del numero de relaciones preferenciales de traducciones entre las lenguas de los países de Europa Central, Europa del Este y los países mediterráneos. La desaparición del serbio-croata que da lugar a dos lenguas nuevas y distintas (el serbio y el croata) es una de las primeras causas de este aumento en los lazos preferenciales de traducción. El croata se conecta con el eslovaco y

\footnotetext{
34 En una correspondencia personal, Abram De Swaan destacaba la especificidad del declive de la lengua rusa: "The dramatic illustration of the decline of Russian (which somewhat contradicts the point that you quote from my book about the persistence of colonial languages long after colonial domination has disappeared". Cf. el pie de página 25 del presente artículo: "It shows how much language constellations are determined by political events, but also how they often survive long after this political base has disappeared"). In this case political and economic decline does appear to have hastened the decline of the language. Like Japanese, apparently it had not been a language imposed by occupation long enough. English and French imperialism after all persisted for a few centuries in Africa and Spanish and English in America so as to permanently undo the language constellations these occupied lands had had before the occupation. Nonetheless, the new language in Eastern Europe is English (much more so than German that had been there long before '39-'45) - an entirely new appearance".

35 El nynorsk (néo-norvégien en francés), llamado landsmål hasta 1929, es una de las dos normas de la lengua escrita noruega. La segunda es el bokmål: que corresponde al noruego en nuestro estudio. El 12 de mayo de 1885, una ley considera las dos lenguas como iguales y en 1980 las dos son reconocidas como lenguas oficiales. El nynorsk se utiliza hoy por aproximadamente un 10 a un $15 \%$ de la población de Noruega. (Fuente: www. wikipedia.org.)
} 
el polonés, mientras que el serbio se relaciona con las lenguas oficiales de los países limítrofes de Serbia (albanés, macedonio, búlgaro, rumano, húngaro), así como con el griego, el ucraniano, el esloveno, el francés y -evidentemente-, con el inglés. Otras relaciones que habían desaparecido después de 1989 reaparecen como es el caso del ucraniano con el polonés y del lituano con el letón. No obstante, esta figura nos muestra que un grupo de lenguas conectadas antes de 1989 por claras razones políticas, dejan de tener relaciones preferenciales de traducción con posterioridad a la caída del muro de Berlín. 


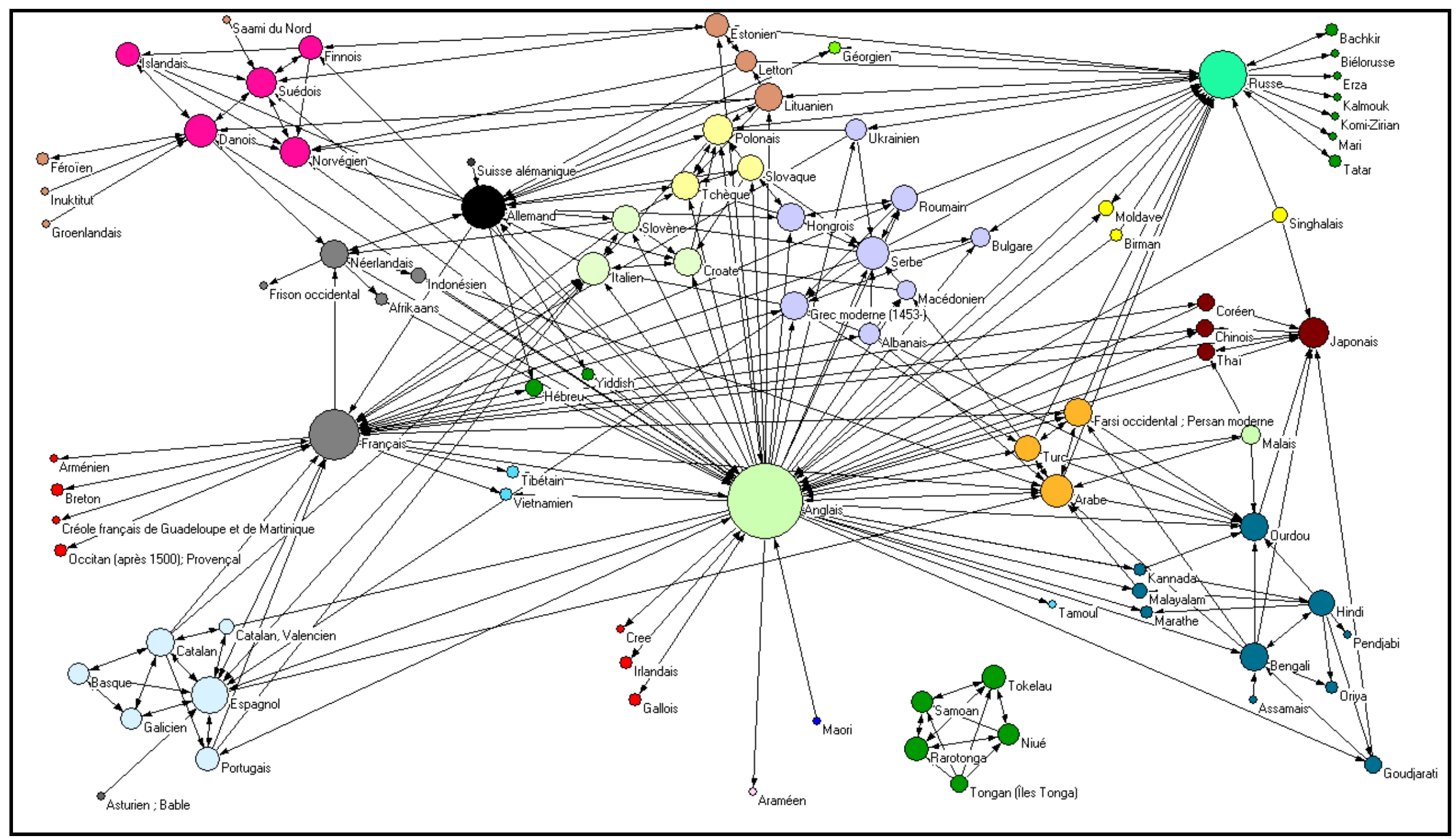

Figura 7. Las relaciones preferenciales de traducción durante el período 1997-2001. 
En contrapartida, se crean nuevos lazos preferenciales: se asiste en particular al refuerzo de las relaciones entre las lenguas de tres países bálticos y las cuatro lenguas de países nórdicos muy cercanos geográficamente. Hasta 1989; el finés era la única lengua conectada con lenguas de países bálticos. En el período 1992-1996, entre las tres lenguas de países bálticos, solo el estonio se relaciona con tres lenguas de países nórdicos. Durante el período 1997-2001, tanto el noruego como el sueco, el finés y el danés, se conectan con al menos una de las tres lenguas de países bálticos.

La figura confirma que casi todas las relaciones preferenciales de traducción del ruso se basan hoy en criterios geográficos y no políticos. El único ejemplo contrario resulta particularmente interesante: la exclusividad de relaciones preferenciales del bielorruso. Esta relación va a contra-corriente de la tendencia internacional que apunta a la creación de lazos preferenciales de traducción con el inglés. El régimen totalitario de Bielorrusia y su comercio exterior enteramente volcado hacia Rusia podrían explicar este resultado.

\section{Otros aspectos a considerar en la explicacion de la dinàmica de flujos de traducciones}

En términos generales, la explicación de la dinámica de flujos de traducciones a partir de los numerosos ejemplos citados precedentemente nos permite comprender la disminución de la diversidad cultural constatada después de 1989. La baja de la densidad de relaciones de traducción se ve ligada a la reconfiguración del "sistema-mundo", cuya dinámica descansa sobre todo en consideraciones políticas y geográficas. En particular éste es el caso de las lenguas situadas en posiciones intermedias, esto es, las 139 lenguas más importantes dejando de lado al inglés y -en menor medida- al francés y al alemán, que conservan un reconocimiento mundial bastante importante.

En el caso de estas tres últimas lenguas, no hay que menospreciar la dimensión económica como explicación de su reconocimiento. Por ejemplo, la hiper-centralización del inglés tiene orígenes políticos pero también económicos (economías en gastos de traducción, ahorro de tiempo; incluso si el dominio del inglés debe mantenerse viendo películas o leyendo obras) ${ }^{36}$. En consecuencia, los editores estarían menos inclinados a traducir textos en lenguas periféricas si existen más lectores en inglés ${ }^{37}$, lo que paradojalmente limita el crecimiento de la proporción de traducciones realizadas a partir

\footnotetext{
36 Ver De Swaan (2001, Pág. 46). El aprendizaje del inglés permite igualmente abrirse a otras opiniones o conocimientos (De Swaan, 2001, Pág.42) y a la publicación directa en inglés (fenómeno particularmente presente en el ámbito de la investigación académica). Parece difícil generalizar este último comentario a todos los tipos de traducciones y en particular a las traducciones en el ámbito literario: en general resulta más fácil escribir una novela en la lengua materna que en inglés.

37 Ver De Swaan (2001, Pág. 46).
} 
del inglés.

Por otro lado, las lenguas de países africanos se mantienen, con raras excepciones, alrededor del grupo de las 526 lenguas menos importantes: la capacidad de producción de estas naciones impide un reconocimiento importante de las lenguas habladas en estos países, descontando las lenguas de los antiguos países colonizadores. Otras consideraciones como el tamaño del país permiten explicar el reconocimiento de algunas lenguas. Este factor interviene en el caso de India: las lenguas indias tienen una gran cantidad de relaciones preferenciales de traducción entre ellas, pero no existe prácticamente ningún lazo preferencial entre las lenguas indias y las lenguas de otros países asiáticos, aparte del árabe y del japonés.

Utilizando el criterio de la relación preferencial, se constata que existe relativamente poca evolución entre los dos últimos períodos estudiados (exceptuando, por supuesto, los cambios relativos a la caída de la ex URSS). Este artículo propone en efecto una visión a largo plazo y a otra escala que aquella de los productores, traductores y lectores: las evoluciones puntuales (menos de cinco años) o más localizadas (relacionadas con un tipo de autor, un tipo de libro, una corriente literaria en particular...etc.) son dejadas de lado.

\section{Conclusión}

Confrontar el concepto de globalización a un estudio empírico constituía nuestro objetivo inicial. La tentativa de síntesis de las definiciones del concepto de globalización no se corresponden con lo que hemos observado, en particular respecto a la integración y la creciente interdependencia: la utilización de este concepto resulta, entonces, inadecuada para nuestro objeto. En efecto, nuestro estudio aporta elementos empíricos sobre la concentración de intercambios culturales y, por consiguiente, sobre la concentración de la producción de bienes culturales, que se ilustra especialmente en una disminución de la densidad de flujos observados, en circunstancias que el número de traducciones se ha casi duplicado a partir de 1989.

Considerando lo anterior: ¿podemos temer una disminución de la diversidad cultural en los próximos años? Si la pregunta permanece abierta, nos parece que ésta se relaciona con la futura dinámica del flujo de traducciones. Ahora bien, este artículo aporta igualmente elementos de reflexión sobre dicho tema. Por ejemplo, hemos podido mostrar que el aumento de la proporción de traducciones realizadas a partir del inglés ha sido muy importante entre 1989 y 1992 mientras que, en paralelo, el reconocimiento del ruso no ha cesado de disminuir. 
La importancia del lugar que ocupan las lenguas, más allá de la calidad de las obras como factor explicativo del reconocimiento de los idiomas, no es una idea nueva. Así, según Pascale Casanova (2002, Pág. 9): «para darse una oportunidad de comprender los verdaderos (y a menudo ignorados) intereses en juego en la traducción de un texto, resulta necesario describir antes la posición que ocupan las lenguas de partida y de llegada en el universo de las lenguas literarias, situar enseguida al autor traducido en el campo literario mundial (...); analizar finalmente la posición de los diversos agentes que participan en el proceso de consagración de la obra". Johan Heilbron (1999) comparte esta constatación ${ }^{38}$, aún si Pascale Casanova (2002) insiste sobre todo en el concepto de dominación ${ }^{39}$. Como Heilbron (1999) lo precisa, por otro lado ${ }^{40}$, es necesario relacionar el reconocimiento de las lenguas a nivel internacional con las estrategias de las editoriales y la dinámica de la economía del libro.

Si compartimos la necesidad de un acercamiento entre estas dos escalas, sin dejar de mantener una visión de largo y corto plazo, este artículo cuestiona la validez de un enfoque del reconocimiento de lenguas fundado únicamente sobre el concepto de dominación. Ciertamente, el inglés es la lengua dominante a nivel internacional: esta lengua ocupa hoy una posición casi hegemónica. Sin embargo, el concepto de dominación difícilmente se puede aplicar a otras lenguas: en efecto, en numerosas ocasiones, pudimos explicar la dinámica de los flujos de traducciones por consideraciones geográficas, sobre todo a partir de 1989.

Más generalmente, este artículo muestra que el proceso de "globalización" no puede ser aprehendido sino mediante trabajos empíricos que utilicen otras herramientas que aquellas utilizadas habitualmente ${ }^{41}$. Así, otras lógicas saldrán a la superficie y aportarán

\footnotetext{
38 « The analysis of this world-system, and the position which various language groups occupy within it, is a precondition for understanding the role of translations in specific local or national contexts. (...) The significance of translations within language groups, for example, is shown to depend primarily on the position of the language within the international system », Heilbron (1999)

39 La idea es cercana al término «periférico» utilizado en el enfoque de Heilbron. Sin embargo, Pascale Casanova aporta una precisión: "Para medir el volumen propio de este capital, propongo transponer al universo literario los criterios utilizados por la sociología política, con la condición de remplazar los términos opuestos "centro/periferia" -que no tienen otra implicación sino espacial o simplemente jerárquica- por la oposición "dominante/dominado", que supone una estructura de dominación y de relaciones de fuerza. Así, no opondremos lenguas centrales a lenguas periféricas, sino que lenguas dominantes a lenguas dominadas, lo que, lejos de ser un simple cambio semántico, transforma la propia perspectiva del análisis y el tipo de instrumentos teóricos movilizados (Casanova, 2002, Pág. 8) »

40 Heilbron (1999): «There is obviously no simple and immediate transition from a world-system analysis to the level of a national publishing industry or the understanding of particular translation strategies. The world-system is concerned with the most general set of conditions, and for a more complete account, it is necessary to link these general conditions to the social dynamics of the publishing business and its different segments ».

41 Miguel Centeno ha reunido numerosos datos utilizando la técnica del Análisis de Redes Sociales sobre diversas temáticas relacionadas con la globalización. Todavía no establecido una síntesis precisa, aún cuando parecería que aquello que constato a propósito de las traducciones (la reducción en la diversidad de flujos) estaría en la línea de lo que él ha observado. Más información en: http://www.princeton.edu/ cenmiga/
} 
en consecuencia una mejor comprensión de los intercambios internacionales.

\section{Bibliografía}

Adorno T.W. et Horkheimer M. (1974) [1947]. La production industrielle de biens culturels in La Dialectique de la raison, Paris, Gallimard.

Allemand Sylvain et Ruano - Borbalan Jean-Claude (2005). La mondialisation, Collection idées reçues, Le cavalier bleu éditions.

Bourdieu Pierre (1979). La distinction, critique sociale du jugement, Les éditions de minuit.

Bourdieu Pierre et Wacquant Loïc (2000). «La nouvelle vulgate planétaire ». Le Monde diplomatique, mai 2000, pp. 6-7.

Cachin Marie-Françoise et Bruyère Claire (2002). « La traduction au carrefour des cultures » in Les mutations du livre et de l'édition dans le monde du 18ème siècle à l'an 2000, sous la direction de Jacques Michon et Jean-Yves Mollier, pp. 506-525.

Casanova Pascale (2002). "Consécration et accumulation de capital littéraire. La traduction comme échange inégal », Les Actes de la recherche en sciences sociales, Septembre 2002, pp. 7-20

Chartier Roger (sous la direction de) (1985). Pratiques de la lecture, Payot et Rivages.

Cuche Denis (1996). La notion de culture en sciences sociales, Paris, La Découverte (Repères 205).

De Saint-Ange Arnault. Le monde de l'édition 2003-2005. La fin d'une époque. Article disponible à l'adresse suivante : .

De Swaan Abram (2001). Words of the World, Cambridge, Polity Press.

Hargittai Eszter et Miguel Angel Centeno (2001). Defining A Global Geography, American Behavioral Scientist, 44 (10)).

Heilbron Johan (1999). "Toward a sociology of translation. Book translations as a cultural world-system, in European Journal of Social Theory », volume 2 (4), pp. 429444.

Heilbron J ohan (2001). «Echanges culturels transnationaux et mondialisation : quelques réflexions ». Regards sociologiques, 22, pp. 141-154.

Horellou - Lafarge \& Segré (2003). Sociologie de la lecture, Paris, La Découverte (Repères 376).

Institut Statistique de I'Unesco. Echanges internationaux d'une sélection de biens et de services culturels, 1994-2003.

Krugman, Paul R. (2000). La mondialisation n'est pas coupable: vertus et limites du libre-échange, Paris, La Découverte.

Lazega Emmanuel (1999). Réseaux sociaux et structures relationnelles, collection Que sais-je ?, numéro 3399, PUF.

Lévi-Strauss Claude (1958). Anthropologie Structurale, Paris, Plon. 
Marcuse Herbert (1968) [1964]. L’homme unidimensionnel, Arguments, Les éditions de Minuit.

Schiffrin André (1999). L'édition sans éditeurs, La fabrique éditions. 